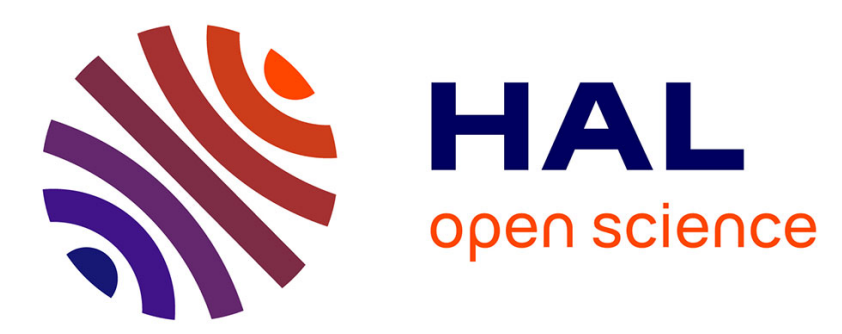

\title{
Distortions of multivariate distribution functions and associated level curves: applications in multivariate risk theory
}

\author{
Elena Di Bernardino, Didier Rullière
}

\section{To cite this version:}

Elena Di Bernardino, Didier Rullière. Distortions of multivariate distribution functions and associated level curves: applications in multivariate risk theory. Insurance: Mathematics and Economics, 2013, 53, pp.190-205. 10.1016/j.insmatheco.2013.05.001 . hal-00750873v4

\section{HAL Id: hal-00750873 \\ https://hal.science/hal-00750873v4}

Submitted on 6 May 2013

HAL is a multi-disciplinary open access archive for the deposit and dissemination of scientific research documents, whether they are published or not. The documents may come from teaching and research institutions in France or abroad, or from public or private research centers.
L'archive ouverte pluridisciplinaire HAL, est destinée au dépôt et à la diffusion de documents scientifiques de niveau recherche, publiés ou non, émanant des établissements d'enseignement et de recherche français ou étrangers, des laboratoires publics ou privés. 


\title{
Distortions of multivariate distribution functions and associated level curves: applications in multivariate risk theory
}

\author{
Elena Di Bernardino ${ }^{1}$, Didier Rullière ${ }^{2}$
}

\begin{abstract}
In this paper, we propose a parametric model for multivariate distributions. The model is based on distortion functions, i.e. some transformations of a multivariate distribution which permit to generate new families of multivariate distribution functions. We derive some properties of considered distortions. A suitable proximity indicator between level curves is introduced in order to evaluate the quality of candidate distortion parameters. Using this proximity indicator and properties of distorted level curves, we give a specific estimation procedure. The estimation algorithm is mainly relying on straightforward univariate optimizations, and we finally get parametric representations of both multivariate distribution functions and associated level curves. Our results are motivated by applications in multivariate risk theory. The methodology is illustrated on simulated and real examples.
\end{abstract}

Keywords: Multivariate probability distortions, Level sets estimation, Iterated compositions, Hyperbolic conversion functions, Multivariate risk measures.

\section{Introduction}

Multivariate distributions can be modeled using parametric marginal distribution functions and parametric copulas, or directly by some specific parametric expressions of the multivariate cumulative distribution function. However some problems of such parametric forms can arise. We underline, for instance, the difficulty (see for example Bienvenüe and Rullière, 2012, in the univariate case)

- to fit non-regular or multimodal distributions using classical unimodal distributions;

- to change the number of parameters and to improve a fit to observed data;

- to estimate the parameters when their number is large;

- to get analytical expressions for level curves of the distribution functions.

In order to overcome these shortcomings, in this paper we define multivariate distributions by using probability distortions. We show that using distortions has several advantages compared to using classical parametric multivariate distributions. In particular we focus on:

- the possibility to get analytical expressions both for the multivariate distribution function and for the associated level-curves;

- the huge variety of reachable distributions (multimodal, etc.), with the possibility to improve the fit by adding parameters (via distortions composition), and the possibility to converge to any target in dimension 1;

\footnotetext{
${ }^{1}$ CNAM, Paris, Département IMATH, 292 rue Saint-Martin, Paris Cedex 03, France, elena.di_bernardino@cnam.fr

${ }^{2}$ Université de Lyon, Université Lyon 1, ISFA, Laboratoire SAF, 50 avenue Tony Garnier, 69366 Lyon, France, rulliere@univ-lyon1.fr
} 
- some estimation facilities, with the possibility to get very good initial values for parameters even with a large number of parameters.

Using parametric distortions will also have some advantages compared to nonparametric fitting procedures like multivariate splines (e.g. see Awanou et al., 2005) or kernel estimation (e.g. see Charpentier et al. 2006). Among advantages of the considered distortions, one can cite the possibility to ensure regular conditions (like monotonicity and positiveness of density function), and the possibility to get analytical expressions for both cumulative distribution function and for level curves.

\section{Applications in multivariate risk theory}

Our results are motivated by applications in multivariate risk theory. In the following we detail the connection between the multivariate level-curves and the recent developments of risk theory.

During the last decades, researchers joined efforts to properly quantify and manage risks. Traditionally, risk measures are thought of as mappings from a set of real-valued random variables to the real numbers. However, it is often insufficient to consider a single real measure to quantify risks created by business activities, especially if the latter are affected by other external risk factors. Consequently we need a generalization of univariate classical risk measures in dimension higher than one. In the recent literature, an intuitive and immediate generalization of the Value-at-Risk measure in the case of a $d$-dimensional loss distribution function $F$ is represented by its $\alpha$-quantile curves, i.e. $\partial L(\alpha)=\left\{\mathbf{x} \in \mathbb{R}_{+}^{d}: F(\mathbf{x})=\alpha\right\}$. Then we define:

$$
\operatorname{VaR}_{\alpha}(F)=\partial L(\alpha), \text { for } \alpha \in(0,1) .
$$

This generalization of the Value-at-Risk may be useful to quantify dependent risks: dependent environmental risks factors in same geographic area, several dependent business lines of a financial institution (e.g. see Chebana and Ouarda, 2011; Cousin and Di Bernardino, 2012). The interest reader is also referred to Tibiletti (1993), Belzunce et al. (2007), Embrechts and Puccetti (2006), Nappo and Spizzichino (2009), Di Bernardino et al. (2011).

In the following we present some interesting applications of $\operatorname{VaR}_{\alpha}(F)$ proposed in the recent literature, to model multivariate risk problems.

Firstly, the estimation of multivariate quantile curves may be applied to several hydrological phenomena, such as droughts, storms and floods. Chebana and Ouarda, 2011 remark that the frequency analysis in hydrology focuses on the estimation of quantities (e.g., flows or annual rainfall) corresponding to a certain return period. For a random variable $X$ that represents the magnitude of an event that occurs at a given time and at a given site, the quantile of order $1-\frac{1}{T}$ expresses the magnitude of the event which is exceeded with a probability equal to $\frac{1}{T}$. $T$ is then called the return period. In the bivariate hydrological event distributions and corresponding return periods have been extensively studied e.g. in Salvadori et al. (2007). For instance, for the event $\{X>x, Y>y\}$, Salvadori et al. (2007) defined the bivariate return period as the positive number $T^{x, y}$ given by:

$$
T^{x, y}=\frac{1}{\mathbb{P}[X>x, Y>y]} .
$$

In Chebana and Ouarda, 2011 the bivariate volume flood and peak flood vector $(X, Y)$ is considered. They estimate the bivariate level curves $\partial L\left(1-\frac{1}{T}\right)$ of the distribution function $F_{(X, Y)}$ (i.e. $\operatorname{VaR}_{1-\frac{1}{T}}(F)$ in Equation (1)) in order to provide the bivariate return period relative to the hydrological phenomenon.

de Haan and Huang (1995) consider the real problem to determinate the height of a projected sea-dike. On the basis of high tide water levels observed during 100 years one has to design the height of the dike in 
such a way that the return period of a flood is 10000 years. The requirement is that the return period of a flood at either one of two places along the coast is 10000 years. This multidimensional risk problem is solved by estimating the levels curves $\partial L(1-\alpha)=\{(x(\alpha), y(\alpha)): F(x(\alpha), y(\alpha))=1-\alpha\}$, of a bivariate distribution function $F$, for $\alpha \in(0,1)$.

Multivariate generalization of Value-at-Risk proposed in (1) is also used in the context of solvency capital allocation for financial institutions with multi-branch businesses confronted to risks with specific characteristics. As remarked by Cousin and Di Bernardino (2012), multivariate quantile curves may be useful to understand how solvency capital requirement (SCR) could be computed in a macro-prudential regulatory framework in which institutions can also be affected by risks undertaken by its competitors. More precisely, let $\mathbf{X}=\left(X_{1}, \ldots, X_{d}\right)$ be a risk vector with $d$-dimensional distribution function $F$ and where, for any $i=1, \ldots, d$, the component $X_{i}$ denotes risk (usually claim or loss) associated with subportfolio $i$. They proposed to consider the multivariate risk measure defined by:

$$
\mathbb{E}[\mathbf{X} \mid \mathbf{X} \in \partial L(\alpha)]
$$

Several properties for the risk measure above have been derived by Cousin and Di Bernardino (2012). In particular, they show that the behavior of this risk measure turns to be consistent with existing properties on univariate risk measures. The multivariate measure $\mathbb{E}[\mathbf{X} \mid \mathbf{X} \in \partial L(\alpha)]$ recalled above is essentially based on a "distributional approach". It is constructed as the conditional expectation of a multivariate random risk vector given that the latter is located in a particular set corresponding to the $\alpha$-level curve of the associated multivariate distribution function $F$. As the total information of the risk portfolio $\mathbf{X}$ is completely described by its multivariate distribution function, the set $\partial L(\alpha)$ captures the information coming both from the marginal distributions and from the multivariate dependence structure. For more details the interested reader is referred to Cousin and Di Bernardino (2012), Di Bernardino et al. (2011), Di Bernardino and Prieur (2012).

Starting from these applications in multivariate risk theory, proposed above, we are interested in parametric representations of multivariate distribution function $F$ and associated multivariate Value-at-Risk $\operatorname{VaR}_{\alpha}(F)$ (i.e. level curves of $F$ ). They may be of great importance in many practical studies of multivariate risks because they analytically describe a multivariate risk-area (see Embrechts and Puccetti, 2006).

Some effort has been made in recent literature to estimate the level-curves of a multivariate distribution function. A non-parametric plug-in estimation of $\operatorname{VaR}_{\alpha}(F)$ is given by Di Bernardino et al. (2011). As introduced above, de Haan and Huang (1995) model a risk-problem of flood using an extremal estimator of $\operatorname{VaR}_{\alpha}(F)$, for $F$ a bivariate distribution function. In this paper we provide an estimation algorithm to estimate multivariate distribution function $F$ and associated $\operatorname{VaR}_{\alpha}(F)$. Differently from the literature cited above, our procedure gives explicit parametric-analytical representations for estimated $F$ and $\operatorname{VaR}_{\alpha}(F)$. This feature can be relevant from a practical point of view.

\section{Organization of the paper}

The paper is organized as follows. In Section 1, we introduce some notations, tools and technical assumptions. In Section 2, we present some transformations of a multivariate distribution function $F$ which permit to generate new families of multivariate distribution functions. In particular, we introduce external distortions (see Section 2.1), and internal ones (see Section 2.2). In Section 3 we propose an estimation procedure in the case of piecewise linear distortions. In Section 4, we deal with estimation in the case of differentiable distortions. Numerical applications are presented in Section 5 using hyperbolic distortion functions. Two real case studies are illustrated in Sections 5.2-5.3. Finally, in Section 5.4 we illustrate some properties of our estimation using simulated data set. 


\section{Basic notions and preliminaries}

Assume that we have $d$ underlying risks described by a $d$-dimensional nonnegative real-valued random vector $\mathbf{X}=\left(X_{1}, \ldots, X_{d}\right)$. Denote its multivariate distribution function by $F: \mathbb{R}_{+}^{d} \rightarrow[0,1]$ with univariate margins $F_{i}\left(x_{i}\right)=P\left(X_{i} \leq x_{i}\right)$, for $i=1, \ldots, d$. Sklar's Theorem (1959) is a well-known result which states that for any random vector $\mathbf{X}$, its multivariate distribution function has the representation

$$
F\left(x_{1}, \ldots, x_{d}\right)=C\left(F_{1}\left(x_{1}\right), \ldots, F_{d}\left(x_{d}\right)\right),
$$

where $C$ is called the copula function. Effectively, it is a distribution function on the $d$-cube $[0,1]^{d}$ with uniform margins and it links the univariate margins to their full multivariate distribution. In the case where we have a continuous random vector, we know that $U_{i}=F_{i}\left(X_{i}\right)$ is an uniform random variable so that we can write

$$
C\left(u_{1}, \ldots, u_{d}\right)=F\left(F_{1}^{-1}\left(u_{1}\right), \ldots, F_{d}^{-1}\left(u_{d}\right)\right),
$$

to be the unique copula associated with $\mathbf{X}$, with quantile functions $F_{i}^{-1}$ defined by:

$$
F_{i}^{-1}(\alpha)=\inf \left\{x \in \mathbb{R}_{+}: F_{i}(x) \geq \alpha\right\}, \quad \text { for } \alpha \in(0,1) .
$$

Denote $\mathcal{F}$ the class of $d$-dimensional distribution functions $F$ such that $F: \mathbb{R}_{+}^{d} \rightarrow[0,1]$ be a continuous partially increasing function ${ }^{3}$ of a non-negative absolutely-continuous random vector ${ }^{4} \mathbf{X}=\left(X_{1}, \ldots, X_{d}\right)$ (with respect to Lebesgue measure $\lambda$ on $\mathbb{R}^{d}$ ). In the following, $F \in \mathcal{F}$ will be said to satisfy regularity conditions.

Let $L(\alpha)=\left\{\mathbf{x} \in \mathbb{R}_{+}^{d}: F(\mathbf{x}) \geq \alpha\right\}$ be the upper $\alpha$-level set of $F$, for $\alpha \in(0,1)$ and $d \geq 2$. Furthermore, for any set $A \subset \mathbb{R}_{+}^{d}$ we denote by $\partial A$ its boundary.

Note that, under the regularity conditions, $\partial L(\alpha)=\left\{\mathbf{x} \in \mathbb{R}_{+}^{d}: F(\mathbf{x})=\alpha\right\}$ has Lebesgue-measure zero in $\mathbb{R}_{+}^{d}$ (e.g., see Property 3 in Tibiletti, 1990). We call $\partial L(\alpha)$ the $\alpha$-level curve of distribution $F$. For instance, if $d=2$, each $\partial L(\alpha)$ for $\alpha \in(0,1)$, is identified by a decreasing curve in the plane $\mathbb{R}_{+}^{2}$ (e.g., see Theorem 2 in Rossi, 1973; Section 2 in Tibiletti, 1991).

We now recall the notion of absolutely monotonic function that will be useful later. The interested reader is referred also to Valdez and Xiao (2011). Assume that $n$ is a non-negative integer.

Definition 1.1 (Absolute monotony) A real function $g(t)$ is said to be absolutely monotonic, of order $n$, on an interval $I$ if the following conditions are satisfied:

- $g$ is continuous on $I$; and

- $g$ has non-negative derivatives of orders up to, and including, $n$, i.e., $g^{(k)}(t) \geq 0$, for all $t$ on the interior of $I$ and for $k=0,1, \ldots, n$.

Remark 1 If $g$ and $h$ are both absolutely monotonic of order $n$ on an interval $I$ and $h$ is defined on $I$ such that $h(t)$ is on the interior of $I$ for all $t$ on the interior of $I$, then the composite function $g \circ h(t)$ is also absolutely monotonic of order $n$ on $I$.

\footnotetext{
${ }^{3} \mathrm{~A}$ function $F\left(x_{1}, \ldots, x_{d}\right)$ is partially increasing on $\mathbb{R}_{+}^{d} \backslash(0)$ if the functions of one variable $g(\cdot)=$ $F\left(x_{1}, \ldots, x_{j-1}, \cdot, x_{j+1}, \ldots, x_{d}\right)$ are increasing. About properties of partially increasing multivariate distribution functions we refer the interested reader to Rossi (1973) and Tibiletti (1991).

${ }^{4}$ We restrict ourselves to $\mathbb{R}_{+}^{d}$ because, in our applications, components of $d$-dimensional vectors correspond to random losses and are then valued in $\mathbb{R}_{+}$. However extensions of our results in the case of multivariate distribution function on the entire space $\mathbb{R}^{d}$ are possible.
} 


\section{Considered distortions}

The aim of this section is to introduce some simple transformations of a multivariate distribution function $F$ which permit to generate new families of multivariate distribution functions. Firstly we will be interested in distortion applied to the function $F$ (called external distortions and leading to a distorted function $\widetilde{F}^{e x t}$ ), secondly we will include also the distortions of marginal components of $F$. In this last case we will finally obtain a global distorted function $\widetilde{F}$. The interested reader is referred, for instance, to Christian Genest in the conference "Distributions with Given Marginals and Statistical Modeling" (Barcelona, July 17-20, 2000) and Durrleman et al. (2000).

Definition 2.1 (Regular external distortions) Let $T:[0,1] \rightarrow[0,1]$ be a continuous and increasing function on the interval $[0,1]$, with $T(0)=0, T(1)=1$, such that $\forall F \in \mathcal{F}, T \circ F \in \mathcal{F}$. Denote by $\mathcal{T}$ the set of such functions $T$.

Proposition 2.1 (A sufficient regular condition) If $T$ is an absolutely monotonic function of order $d$ on the interval $[0,1]$, such that $T(0)=0$ and $T(1)=1$, then $T \in \mathcal{T}$.

Proof: Let $F \in \mathcal{F}$. If $T$ is absolutely monotonic function on the interval $[0,1]$ then in particular $T$ is continuous and increasing. So from Definition 3.6 (using the absolutely monotonic property) and 3.4 (using the continuous and increasing property) in Valdez and Xiao (2011) we obtain that $T \circ F \in \mathcal{F}$.

We remark that assumptions of Proposition 2.1 are sufficient, but not necessary. The interested reader is also referred to Durante et al. (2010).

\subsection{External distortion}

In this section we introduce the external distorted multivariate distribution function $\widetilde{F}^{\text {ext }}$.

Definition 2.2 (Externally distorted distribution) Let $F \in \mathcal{F}$ a d-dimensional distribution function that satisfies the regularity conditions. If $T \in \mathcal{T}$ we define the external distorted multivariate distribution function by $\widetilde{F}^{\text {ext }}(\boldsymbol{x})=T \circ F(\boldsymbol{x})$ with $\boldsymbol{x} \in \mathbb{R}_{+}^{d}$.

Furthermore we introduce the following distortion for the upper $\alpha$-level set of $F$.

Proposition 2.2 (External distortion for upper $\alpha$-level set) Let $\alpha \in(0,1)$ and $L(\alpha)=\left\{\boldsymbol{x} \in \mathbb{R}_{+}^{d}\right.$ : $F(\boldsymbol{x}) \geq \alpha\}$. If $T \in \mathcal{T}$, it holds that:

$$
\widetilde{L}^{e x t}(\alpha)=\left\{\boldsymbol{x} \in \mathbb{R}_{+}^{d}: \widetilde{F}^{e x t}(\boldsymbol{x}) \geq \alpha\right\}=L\left(T^{-1}(\alpha)\right) .
$$

Proof: Since $\widetilde{F}^{e x t}(\mathbf{x})=T \circ F(\mathbf{x})$ and $T$ is invertible in $[0,1]$ then

$$
\widetilde{L}^{e x t}(\alpha)=\left\{\mathbf{x} \in \mathbb{R}_{+}^{d}: T \circ F(\mathbf{x}) \geq \alpha\right\}=\left\{\mathbf{x} \in \mathbb{R}_{+}^{d}: F(\mathbf{x}) \geq T^{-1}(\alpha)\right\} .
$$

Hence the result.

Remark 2 If $T$ is absolutely monotonic so that it fulfils sufficient assumptions of Proposition $2.1, T^{-1}$ is a concave function. Then in particular $\alpha:=T^{-1}(\beta)>\beta$, for $\beta \in(0,1)$. This means the transformation $T$ transforms the upper $\beta$-level set of $F$ into the upper $\alpha$-level set, with higher risk level $\alpha$.

Furthermore, we remark that using an external distortion we are able to modify the dependence structure of the random vector $\mathbf{X}$. Indeed, for simplicity in the case $d=2$, we easily obtain the following property: 
Remark 3 (Dependence impact of external distortion) Let $X$ and $Y$ be two independent real random variables (i.e., $\left.F(x, y)=F_{X}(x) F_{Y}(y)\right)$ and $T$ be a distortion such that $T \in \mathcal{T}$. Let $(\tilde{X}, \tilde{Y})$ be a vector with distribution function $\widetilde{F}^{e x t}(x, y)=T \circ F(x, y)$. Since in general $T \circ\left(F_{X}(x) F_{Y}(y)\right) \neq$ $\left(T \circ F_{X}(x)\right) \cdot\left(T \circ F_{Y}(y)\right), \tilde{X}$ and $\tilde{Y}$ are not necessarily independent.

\subsection{Global distortion}

We now include to the externally distorted distribution $\widetilde{F}^{e x t}$ also the distortions of marginal components of $F$. In this last case we will finally obtain a global distorted distribution function $\widetilde{F}$.

Definition 2.3 (Distorted distribution) Let $F$ be a d-dimensional distribution function. Let $T \in \mathcal{T}$ and $T_{i}:[0,1] \rightarrow[0,1]$ be a continuous non-decreasing function, such that $T_{i}(0)=0, T_{i}(1)=1$, for $i=1, \ldots, d$. We introduce the global distorted distribution function $\tilde{F}$ of $F$ as :

$$
\tilde{F}\left(x_{1}, \ldots, x_{d}\right)=T \circ C\left(T_{1}^{-1} F_{1}\left(x_{1}\right), \ldots, T_{d}^{-1} F_{d}\left(x_{d}\right)\right) .
$$

The interested reader is also referred to Valdez and Xiao (2011) (Definitions 3.2 and 3.6) and Charpentier (2008). They deal with the particular case $T=T_{1}=\ldots=T_{d}$.

Proposition 2.3 (Distorted margins) Denote by $\tilde{F}_{1}, \ldots \tilde{F}_{d}$ the marginal distributions of the joint distribution $\tilde{F}$. Then,

$$
\tilde{F}_{i}=T \circ T_{i}^{-1} \circ F_{i}, \text { for } i=1, \ldots, d .
$$

In analogy with Proposition 2.2, we now analyze the impact of the global distortion on the $\alpha$-level curves of $F$.

Proposition 2.4 (Distorted $\alpha$-level curves) Let $F$ be a d-dimensional distribution function and $\widetilde{F}$ be as in Definition 2.3. Let $\partial \widetilde{L}(\alpha)=\left\{\boldsymbol{x} \in \mathbb{R}_{+}^{d}: \widetilde{F}(\boldsymbol{x})=\alpha\right\}$. A parametric expression for this distorted $\alpha$-level curve is:

$$
\partial \tilde{L}(\alpha)=\left\{\left(F_{1}^{-1} \circ T_{1}\left(u_{1}\right), \ldots, F_{d}^{-1} \circ T_{d}\left(u_{d}\right)\right),\left(u_{1}, \ldots, u_{d}\right) \in(0,1)^{d}, C\left(u_{1}, \ldots, u_{d}\right)=T^{-1}(\alpha)\right\} .
$$

Or equivalently, as a function of marginal distorted distributions $\widetilde{F}_{i}, i=1, \ldots, d$ :

$$
\partial \widetilde{L}(\alpha)=\psi_{T}\left(\partial L\left(T^{-1}(\alpha)\right)\right), \quad \forall \alpha \in(0,1),
$$

with

$$
\psi_{T}(\boldsymbol{x})=\left(\widetilde{F}_{1}^{-1} \circ T \circ F_{1}\left(x_{1}\right), \ldots, \widetilde{F}_{d}^{-1} \circ T \circ F_{d}\left(x_{d}\right)\right),
$$

where the function $\psi_{T}: \mathbb{R}^{d} \rightarrow \mathbb{R}^{d}$ and $\boldsymbol{x}=\left(x_{1}, \ldots, x_{d}\right) \in \mathbb{R}^{d}$.

Proof: It sufficient to remark that

$\widetilde{L}(\alpha)=\left\{\left(x_{1}, \ldots x_{d}\right): C\left(F_{1}\left(x_{1}^{\prime}\right), \ldots, F_{d}\left(x_{d}^{\prime}\right)\right) \geq T^{-1}(\alpha), F_{1}\left(x_{1}^{\prime}\right)=T_{1}^{-1} \circ F_{1}\left(x_{1}\right), \ldots, F_{d}\left(x_{d}^{\prime}\right)=T_{d}^{-1} \circ F_{d}\left(x_{d}\right)\right\}$.

Hence

$$
\partial \widetilde{L}(\alpha)=\psi_{T_{1}, \cdots, T_{d}}\left(\partial L\left(T^{-1}(\alpha)\right)\right), \quad \forall \alpha \in(0,1),
$$

where $\psi_{T_{1}, \cdots, T_{d}}: \mathbb{R}^{d} \rightarrow \mathbb{R}^{d}$,

$$
\mathbf{x} \mapsto \psi_{T_{1}, \cdots, T_{d}}(\mathbf{x})=\left(F_{1}^{-1} \circ T_{1} \circ F_{1}\left(x_{1}\right), \ldots, F_{d}^{-1} \circ T_{d} \circ F_{d}\left(x_{d}\right)\right),
$$


with $\mathbf{x}=\left(x_{1}, \ldots, x_{d}\right) \in \mathbb{R}^{d}$. For Equation (2) one can write:

$$
\begin{aligned}
\partial \tilde{L}(\alpha) & =\psi_{T_{1}, \ldots, T_{d}}\left(\partial L\left(T^{-1}(\alpha)\right)\right) \\
& =\left\{\left(F_{1}^{-1} \circ T_{1} \circ F_{1}\left(x_{1}\right), \ldots, F_{d}^{-1} \circ T_{d} \circ F_{d}\left(x_{d}\right)\right),\left(x_{1}, \ldots, x_{d}\right) \in \partial L\left(T^{-1}(\alpha)\right)\right\} \\
& =\left\{\left(F_{1}^{-1} \circ T_{1} \circ F_{1}\left(x_{1}\right), \ldots, F_{d}^{-1} \circ T_{d} \circ F_{d}\left(x_{d}\right)\right), C\left(F_{1}\left(x_{1}\right), \ldots, F_{d}\left(x_{d}\right)\right)=T^{-1}(\alpha)\right\} \\
& =\left\{\left(F_{1}^{-1} \circ T_{1}\left(u_{1}\right), \ldots, F_{d}^{-1} \circ T_{d}\left(u_{d}\right)\right),\left(u_{1}, \ldots, u_{d}\right) \in(0,1)^{d}, C\left(u_{1}, \ldots, u_{d}\right)=T^{-1}(\alpha)\right\} .
\end{aligned}
$$

For Equation (3), the result comes down from Propositions 2.3 and Equation (5).

If initial copula $C$ can be inverted, that is $\left\{\left(u_{1}, \ldots, u_{d}\right) \in(0,1)^{d}, C\left(u_{1}, \ldots, u_{d}\right)=T^{-1}(\alpha)\right\}$ is given, and if $F_{1}, \ldots, F_{d}$ admit analytic expressions for inverse distributions then Equation (2) can be particular useful to get an analytic expression for $\alpha$-level curves.

Proposition 2.5 (Distorted copula) Denote $\widetilde{C}\left(u_{1}, \ldots, u_{d}\right)$ the distorted copula such that $\tilde{F}\left(x_{1}, \ldots, x_{d}\right)=$ $\widetilde{C}\left(\widetilde{F}_{1}\left(x_{1}\right), \ldots, \widetilde{F}_{d}\left(x_{d}\right)\right)$. Then copula $\tilde{C}$ only depends on external distortion $T$, i.e.

$$
\tilde{C}\left(u_{1}, \ldots, u_{d}\right)=T\left(C\left(T^{-1}\left(u_{1}\right), \ldots, T^{-1}\left(u_{d}\right)\right)\right) .
$$

Proof: The result comes down from Proposition 2.3.

Proposition 2.6 (Regular condition in bivariate case) Denote by $C$ the initial copula. In the bivariate case, assume $C$ is twice differentiable w.r.t. $x$ and $y$. Write $C_{x}(x, y)=\frac{\partial}{\partial x} C(x, y), C_{y}(x, y)=\frac{\partial}{\partial y} C(x, y)$ and $C_{x y}(x, y)=\frac{\partial}{\partial x} \frac{\partial}{\partial y} C(x, y)$. Assume $T$ is a continuous and increasing function, and that $T$ is differentiable with respective first and second order derivatives $T^{\prime}$ and $T^{\prime \prime}$. Then $T \in \mathcal{T}$ if and only if:

$$
T^{\prime}(C(x, y)) C_{x y}(x, y)+T^{\prime \prime}(C(x, y)) C_{x}(x, y) C_{y}(x, y) \geq 0, \quad \text { for all } x, y \in[0,1] .
$$

In particular, this condition is satisfied if $T$ is absolutely monotonic of order 2. In the case $C(x, y)=x y$ and this condition becomes:

$$
T^{\prime}(z)+z T^{\prime \prime}(z) \geq 0, \quad \text { for all } z \in[0,1] .
$$

Proof: Write $\tilde{C}(u, v)=T\left(C\left(T^{-1}(u), T^{-1}(v)\right)\right)$. Asking that $\frac{\partial}{\partial u} \frac{\partial}{\partial v} \tilde{C}(u, v) \geq 0$, the condition holds.

This last condition in Equation (6) obviously holds when $T$ is absolutely monotonic of order 2, i.e. when $T^{\prime}(x)$ and $T^{\prime \prime}(x)$ are positive functions of $x$. However, this condition also holds in some cases where $T(x)$ is not a convex function of $x$. This theoretical aspect will be illustrated in Section 5 .

\subsection{Impact of the choice of the initial copula}

The external distortion $T$ induces a distortion of the dependence structure. The distorted copula is given by Proposition (2.5),

$$
\tilde{C}\left(u_{1}, \ldots, u_{d}\right)=T \circ C\left(T^{-1}\left(u_{1}\right), \ldots, T^{-1}\left(u_{d}\right)\right)
$$

The class of reachable copulas does obviously rely on the initial copula $C$. We first remark, as in [13], that if $C\left(u_{1}, \ldots, u_{d}\right)$ is an Archimedean copula with generator $\phi$, that is, for a given suitable generator $\phi$,

$$
C\left(u_{1}, \ldots, u_{d}\right)=\phi^{-1}\left(\phi\left(u_{1}\right)+\ldots+\phi\left(u_{d}\right)\right),
$$

then if $T$ is an admissible distortion, $\tilde{C}$ is an Archimedean copula with distorted generator $\tilde{\phi}=T \circ \phi^{-1}$. From McNeil and Nešlehová (2009), $T$ is a admissible distortion if and only if this distorted generator $T \circ \phi^{-1}$ is a $d$-monotone function (see Definition 2.3 in McNeil and Nešlehová, 2009). In particular in the 
case of a distorted bivariate independent copula the distorted generator $T\left(\mathrm{e}^{-t}\right)$ has to be a $2-$ monotone function. Since $T$ is increasing, this condition is exactly Equation (6) stated above.

In order to study the impact of the choice of the initial copula in our procedure, one can show that:

- Assume in dimension $d=2$ that $C$ is lower Fréchet-Hoeffding (counter-monotonic), $C\left(u_{1}, u_{2}\right)=$ $\max \left\{u_{1}+u_{2}-1,0\right\} . C$ is an Archimedean copula, and $\tilde{C}$ is Archimedean. When $T(x)=x^{p}$, with $p>1$, then $\tilde{C}$ is a Clayton Copula $\tilde{C}\left(u_{1}, u_{2}\right)=\max \left\{0,\left(u_{1}^{-\theta}+u_{2}^{-\theta}-1\right)^{-\frac{1}{\theta}}\right\}$, where $\theta=-\frac{1}{p}$ belongs to $[-1,0)$.

- Assume $C$ is an independent copula: $C\left(u_{1}, \ldots, u_{d}\right)=u_{1} \ldots u_{d} . C$ is an Archimedean copula, and $\tilde{C}$ is an Archimedean copula. In the particular case where $T(x)=x^{p}, p>0, \tilde{C}$ is still an independent copula.

- If $C$ is upper Fréchet-Hoeffding (comonotonic), i.e. $C\left(u_{1}, \ldots, u_{d}\right)=\min \left(u_{1}, \ldots, u_{d}\right)$ then since $T$ is increasing, $\tilde{C}$ is comonotonic. The choice of $T$ does not impact the dependence structure in this case.

\section{Estimation}

Here, we aim at estimating a target-level curve of a global distorted $d$-dimensional distribution function. Firstly we consider the estimation of the internal distortions. If target marginal distributions $\tilde{F}_{i}$, for $i=1, \ldots, d$, are given then the internal distortions can be easily deduced from the external one $T$.

\subsection{Methodology}

Estimation of internal distortions

As a consequence of Proposition 2.3, when target distorted marginal distributions $\tilde{F}_{i}$ are known, and when the global distortion $T$ is given, we have:

$$
T_{i}=F_{i} \circ \tilde{F}_{i}^{-1} \circ T, \quad \text { for } i=1, \ldots, d .
$$

It follows that the main problem in our procedure is to estimate the external distortion $T$.

\section{Estimation of external distortion}

We now aim at estimating the external distortion $T$. When margins are perfectly fitted to given targets $\widetilde{F}_{i}, i=1, \ldots, d$, using Equation (3), the level-curves only depend on the external distortion $T$. For this reason we will denote in the following $\partial \widetilde{L}_{T}(\beta)$ the distorted $\beta$ level-curve:

$$
\partial \widetilde{L}_{T}(\beta)=\psi_{T}\left(\partial L\left(T^{-1}(\beta)\right)\right), \quad \forall \beta \in(0,1),
$$

where the function $\psi_{T}: \mathbb{R}^{d} \rightarrow \mathbb{R}^{d}$,

$$
x \mapsto \psi_{T}(x)=\left(\widetilde{F}_{1}^{-1} \circ T \circ F_{1}\left(x_{1}\right), \ldots, \widetilde{F}_{d}^{-1} \circ T \circ F_{d}\left(x_{d}\right)\right),
$$

with $x=\left(x_{1}, \ldots, x_{d}\right) \in \mathbb{R}^{d}$.

Then, we can summarize some key-points for the estimation of the external distortion $T$ :

- The distorted $\beta$ level-curve $\partial \widetilde{L}_{T}(\beta)$ only depends on the external distortion $T$.

- Finding a good distortion $T$ is strongly related to a good choice of level $\alpha:=T^{-1}(\beta)$. 
- Then we need an indicator of the quality of a candidate level-curve.

Following these considerations, firstly we define a suitable proximity indicator (PI) (see Section 3.2), that quantifies if a candidate level curve is good or not. An algorithm with a practical fit of these distortions in the case of piecewise linear external distortion is given in Section 3.3.

\subsection{A suitable proximity indicator (PI)}

Let $\mathbf{X}^{*}$ a $d$-dimensional random vector with distribution $F^{*}$ that satisfies regularity conditions. Let $\partial L^{*}(\alpha)$ be the $\alpha$-level curve of $F^{*}$. Frequently $\alpha$-level curve $\partial L^{*}(\alpha)$ are difficult to obtain analytically. For this reason we will try to obtain quantities relying on $F^{*}$ rather than $\partial L^{*}$.

In order to evaluate the proximity of a "candidate set" $\gamma$ to the target-curve $\partial L^{*}(\alpha)$ we introduce the following proximity indicator:

$$
P I_{F^{*}, \alpha}(\gamma)=\mathbb{E}_{\mathbf{X}^{*}}\left[\left(F^{*}\left(\mathbf{X}^{*}\right)-\alpha\right)^{2} \mid \mathbf{X}^{*} \in \gamma\right]
$$

Trivially, if $\gamma \subseteq \partial L^{*}(\alpha)$, then $P I_{F^{*}, \alpha}(\gamma)=0$. As an interesting property we point out that the integral expression of formula (9) only depends on the analytical expression of $F^{*}$ and not on $\partial L^{*}(\alpha)$. For an illustration of the proximity indicator in (9) the interested reader is remanded to Example 1 below.

Example 1 (An explicit proximity indicator in a bivariate setting) Let uniform marginal distributions in $[0,1]$ and the bivariate Farlie-Gumbel-Morgenstern copula structure, i.e., $F^{*}(x, y)=x y+$ $\theta x y(1-x)(1-y)$, where $\theta \in[-1,1]$ (see Nelsen, 1999, Example 3.12). Let the "candidate set" be $\gamma_{\alpha}=\left\{(x, y) \in[0,1]^{2}: x y=\alpha\right\}$, for $\alpha \in(0,1)$. In this case we can obtain a closed formula for $P I_{F^{*}, \alpha}(\gamma)$, that depends only to $\theta \in[-1,1]$ and $\alpha \in(0,1)$.

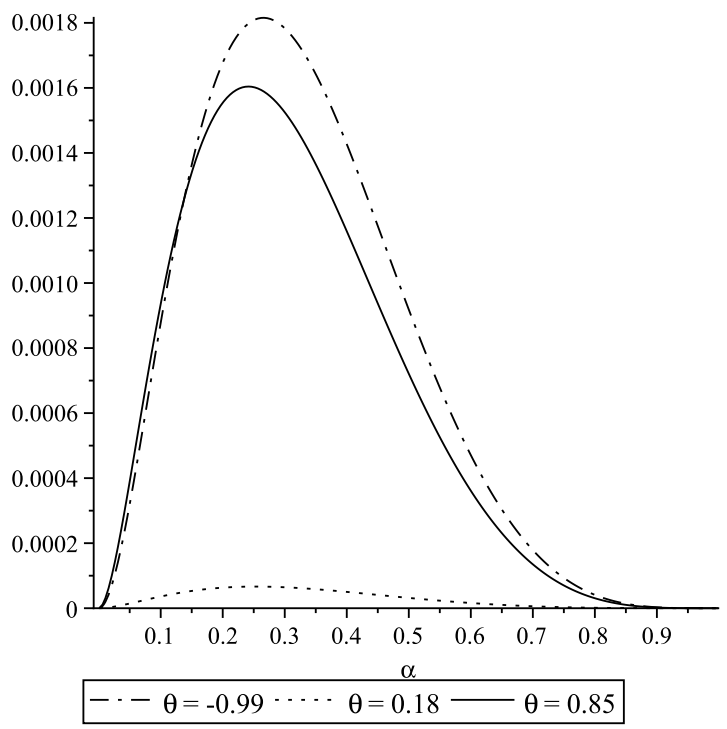

Figure 1: $P I_{F^{*}, \alpha}(\gamma)$, for $\theta=-0.99$ (dashed line), $\theta=0.18$ (dotted line), $\theta=0.85$ (full line), with $F^{*}(x, y)=x y+$ $\theta x y(1-x)(1-y)$ and $\gamma=\left\{(x, y) \in[0,1]^{2}: x y=\alpha\right\}$.

Recall that Farlie-Gumbel-Morgenstern copula reduces to the independence case when $\theta=0$, and it can only model relatively weak dependence in the two extreme cases $(\theta= \pm 1)$ (e.g. Example 3.12, Nelsen, 
1999). Then in particular $P I_{F^{*}, \alpha}(\gamma)=0$, for $\theta=0$. In this sense, Figure 1 shows that $P I_{F^{*}, \alpha}(\gamma)$ takes small values for $\theta$ close to zero $(\theta=0.18)$. Conversely for $\theta$ close to $\pm 1(\theta=-0.99$ and $\theta=0.85)$ we have a greater range of values of $P I_{F^{*}, \alpha}(\gamma)$. Furthermore we remark that $P I_{F^{*}, \alpha}(\gamma)=0$ for $\alpha=0$ and $\alpha=1$, $P I_{F^{*}, \alpha}(\gamma)>0$ for all $\alpha \in(0,1)$ and it is a continuous function with respect to $\alpha$ (see Figure 1 ).

In the case where we do not dispose of an explicit formula for the proximity indicator of a candidate set, we have to estimate it. If $F^{*}$ is supposed to be unknown one can propose the estimated version of the proximity indicator in (9) as:

$$
\widehat{P I}_{F^{*}, \alpha}(\gamma)=P I_{F^{*}, \alpha}(\gamma)
$$

where $\widehat{F^{*}}$ is a consistent smooth estimator of $F^{*}$ admitting a density function. For instance given a $d$-dimensional sample $\left\{x_{1}^{*}, \ldots, x_{n}^{*}\right\}$, with corresponding empirical distribution function $F^{*}{ }_{n}$, the smooth estimator $\widehat{F^{*}}$ could be $f_{K} * F^{*}{ }_{n}$, where $f_{K}$ is a suitable kernel density function (e.g. see Chacón and Rodríguez-Casal, 2010). Furthermore if $\gamma$ is a discrete set of points, then one can write :

$$
\widehat{P I}_{F^{*}, \alpha}(\gamma)=\frac{\sum_{i=1}^{n} \sum_{x \in \gamma}\left(\widehat{F}^{*}(x)-\alpha\right)^{2} f_{K}\left(\frac{x-x_{i}^{*}}{h}\right)}{\sum_{i=1}^{n} \sum_{x \in \gamma} f_{K}\left(\frac{x-x_{i}^{*}}{h}\right)},
$$

In order to build a discrete candidate set from parametric level curve, we introduce following notation that will be useful for numerical algorithms.

Definition 3.1 (Discretization of a level curve) We denote by $\partial L_{g}(\alpha)$ any finite subset of $\partial L(\alpha)$ :

$$
\partial L_{g}(\alpha)=\left\{\left(F_{1}^{-1}\left(\alpha_{1}\right), \ldots, F_{d}^{-1}\left(\alpha_{d}\right)\right),\left(\alpha_{1}, \ldots, \alpha_{d}\right) \in C_{g}^{-1}(\alpha)\right\} .
$$

where $C_{g}^{-1}(\alpha)$ is a finite subset of $\left\{\left(\alpha_{1}, \ldots, \alpha_{d}\right) \in(0,1)^{d}: C\left(\alpha_{1}, \ldots, \alpha_{d}\right)=\alpha\right\}$. In the independence case, one can propose for example, with $g \in \mathbb{N}^{*}$ :

$$
\begin{gathered}
\partial L_{g}(\alpha)=\left\{\left(F_{1}^{-1}\left(\alpha^{p_{1}}\right), \ldots, F_{d}^{-1}\left(\alpha^{p_{d}}\right)\right),\left(p_{1}, \ldots, p_{d}\right) \in P_{g}\right\}, \\
\text { with } P_{g}=\left\{\left(\frac{i_{1}}{g+1}, \ldots, \frac{i_{d}}{g+1}\right),\left(i_{1}, \ldots, i_{d}\right) \in\{1, \ldots, g\}^{d}, i_{1}+\ldots+i_{d}=g+1\right\} .
\end{gathered}
$$

The set $P_{g}$ is a discrete subset of the unit simplex in $(0,1)^{d}$. Since components of its vectors are summing to one, this ensures $\partial L_{g}(\alpha) \subset \partial L(\alpha)$.

\subsection{Estimation algorithm}

Assume that $T$ has a parametric form and belongs to the class of regular distortions $\mathcal{T}$. The best fitted distorted curve for level $\beta$ can be defined as

$$
\partial \widetilde{L}^{o p t}(\beta)=\partial \widetilde{L}_{T^{o p t}}(\beta), \text { with } T^{o p t}=\operatorname{arginf}_{T \in \mathcal{T}} P I_{F^{*}, \beta}\left[\partial \widetilde{L}_{T}(\beta)\right],
$$

and using (8), we get

$$
\partial \widetilde{L}^{o p t}(\beta)=\psi_{T}\left(\partial L\left(T^{-1}(\beta)\right)\right), \text { with } T=\operatorname{arginf}_{T \in \mathcal{T}} P I_{F^{*}, \beta}\left[\psi_{T}\left(\partial L\left(T^{-1}(\beta)\right)\right)\right]
$$

If $T$ has a lot of parameters, the optimization procedure have to face a dimensionality problem (cf. Richard E. Bellman, the curse of dimensionality). Here, the problem comes from the fact that $\psi_{T}$ depends on the searched distortion $T$. 
Assume that at a step $\nu$ of the algorithm, we have a suitable approximation $\psi_{T^{\nu}}$ of $\psi_{T}$, where $T^{\nu}$ is a known distortion. One can define at this step $\nu$ the corresponding fitted level-curve:

$$
\begin{aligned}
\partial{\widetilde{L_{\nu}}}^{\text {opt }}(\beta) & =\psi_{T^{\nu}}\left(\partial L\left(T^{-1}(\beta)\right)\right), \text { with } T=\operatorname{arginf}_{T \in \mathcal{T}} P I_{F^{*}, \beta}\left[\psi_{T^{\nu}}\left(\partial L\left(T^{-1}(\beta)\right)\right)\right] \\
& =\psi_{T^{\nu}}\left(\partial L\left(\alpha^{\nu}\right)\right), \text { with } \alpha^{\nu}=\operatorname{arginf}_{a \in(0,1)} P I_{F^{*}, \beta}\left[\psi_{T^{\nu}}(\partial L(a))\right] .
\end{aligned}
$$

The optimization in Equation (12) is far more easier to solve because it is a one-dimensional optimization, whereas optimization in Equation (11) is an optimization relying on all parameters of $T$.

This trick simply rely on the fact when $\psi_{T^{\nu}}$ is known, finding the best distortion $T$ is equivalent to find the best level $\alpha^{\nu}$, simply by setting $\alpha^{\nu}=T^{-1}(\beta)$. As a consequence, the distortion $T$ is then assumed to pass through the point $\left(\alpha^{\nu}, \beta\right)$.

As a summary, given chosen distorted levels $\beta_{j}$, we aim at finding optimal corresponding levels $\alpha_{j}$, and thus the optimal distortion $T$ passing through of all points $\left(\alpha_{j}, \beta_{j}\right), j=1, \ldots, m$. Such a distortion passing through all points of a set $\Omega$ will be written $\bar{T}_{\Omega}$, as detailed in following definition.

Definition 3.2 (Piecewise linear distortion) Let $\Omega=\left\{\omega_{1}, \ldots, \omega_{m}\right\}, \omega_{j} \in(0,1)^{2}, j=1, \ldots, m, m \in$ $\mathbb{N}^{*}$. We denote by $\bar{T}_{\Omega}(x)$ a given nonparametric function linking point $(0,0)$, points of $\Omega$, and point $(1,1)$. Typically, $\bar{T}_{\Omega}(x)$ can be piecewise linear, or piecewise linear after a change of scale.

Starting from these considerations and Equation (12), we can now introduce the procedure of estimation of $\bar{T}_{\Omega}(x)$, detailed in Algorithm 1.

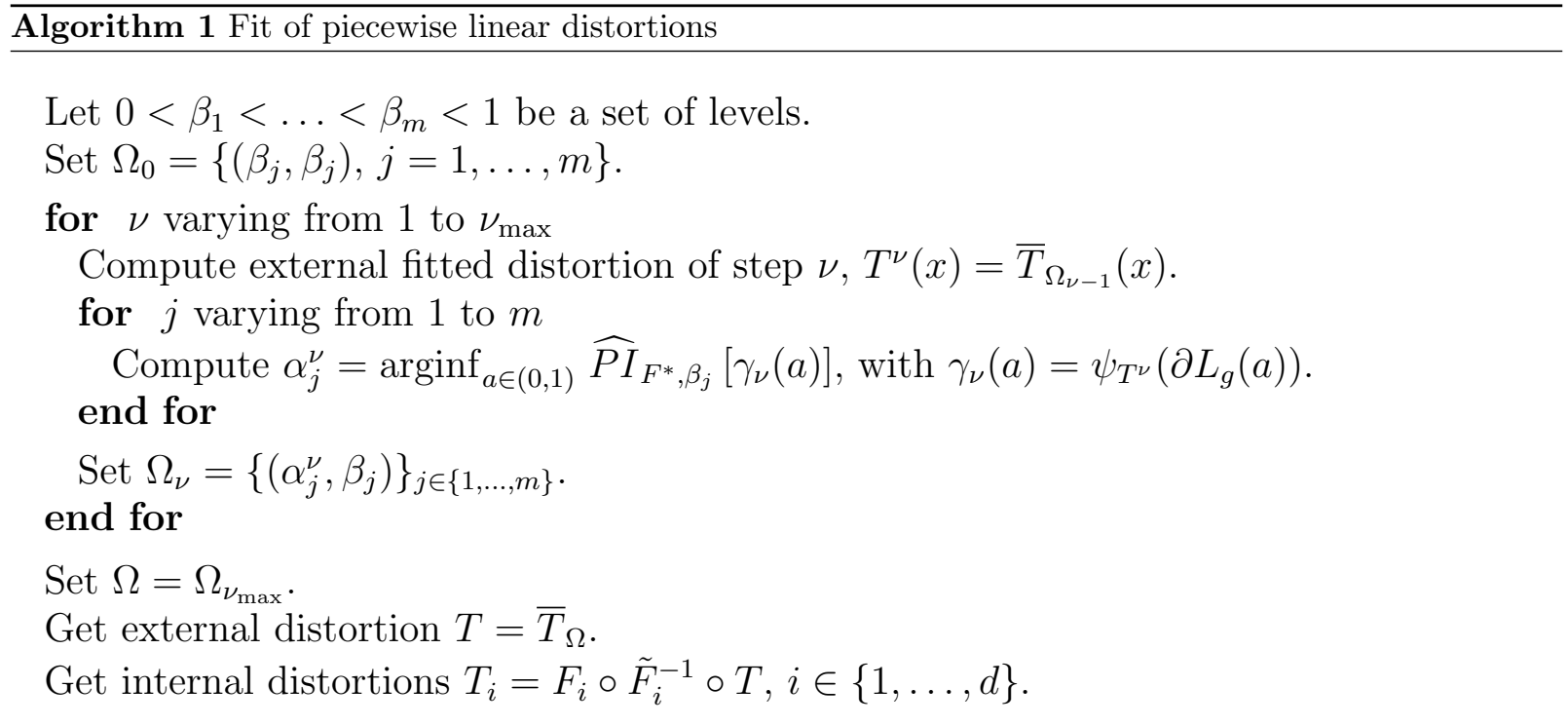

A necessary condition for this algorithm to be applied is that $\bar{T}_{\Omega}(x)$ is an increasing function for $x$, that is for all $i \in\{1, \ldots, m\}, \alpha_{i}^{\nu} \geq \alpha_{i-1}^{\nu}$, where $\alpha_{0}^{\nu}=0$ and $\nu=\nu_{\max }$. This algorithm is motivated by the following consideration. In the case where the levels of $\Omega_{\nu}$ converge to the respective levels of a set $\Omega$, 
then the distortion $T_{\nu}$ converges toward a distortion $T$.

To summarize, Algorithm 1 gives a fitted nonparametric external distortion $\bar{T}$, and corresponding fitted internal distortions $T_{1}, \ldots, T_{d}$.

Remark 4 We summarize here comments about Algorithm 1:

1. From Equations (4) and (10) we have

$$
\gamma_{\nu}(a)=\psi_{T^{\nu}}\left(\partial L_{g}(a)\right)=\left\{\widetilde{F}_{1}^{-1} \circ T\left(\alpha_{1}\right), \ldots, \widetilde{F}_{d}^{-1} \circ T\left(\alpha_{d}\right), \text { with }\left(\alpha_{1}, \ldots, \alpha_{d}\right) \in C_{g}^{-1}(\alpha)\right\},
$$

(see Definition 3.1). Then calibration of external distortion $T$ in Algorithm 1 does not depend on the choice of initial marginal distributions. Indeed in Proposition 2.5 the distorted copula $\widetilde{C}$ is only related to $T$. From another point of view, Algorithm 1 aims at fitting $\widetilde{C}$ with the empirical copula $\widehat{C}$.

2. Furthermore, for each choice of the initial marginals $F_{1}, \ldots, F_{d}$, we can directly derive an expression of the marginal distortions $T_{1}, \ldots, T_{d}$. We will see in Section 4.3 that, using some particular hyperbolic parameterizations, we can fit these distortions as precisely as desired. This convergence property is proved in Bienvenüe and Rullière (2012), Theorem 2. In the case of heavy tail marginal distributions Proposition 3 in Bienvenüe and Rullière (2012) shows that distortions can easily change the tail index of the distribution, in order to explore different tail behaviors.

Remark 5 We summarize here some advantages of Algorithm 1:

1. Using this procedure our $d$-dimensional problem is decomposed in a sequence of univariate optimization problems. We just have to optimize separately $m$ univariate parameters $\alpha_{i}$, corresponding to the levels of the level-curves.

2. Once distortions $\bar{T}, T_{1}, \ldots, T_{d}$ estimated, one can provide analytical expressions for both $\widetilde{F}$ and $\widetilde{L}$. This is an interesting aspect of the procedure above because, frequently, the analytical expression of $\widetilde{L}$ is quite difficult to obtain.

3. Algorithm 1 gives a non parametric estimation of $\bar{T}$ and corresponding internal distortions $T_{1}, \ldots, T_{d}$. Smoothed parametric version of these distortions will be easy to find (see Section 4.3).

In order to get smooth fitted distortions satisfying regular conditions, we propose hereafter some numerical applications using hyperbolic distortions.

\section{Smooth estimation}

\subsection{A particular class of distortion functions}

We take back from Bienvenüe and Rullière (2012) the following key notion of conversion function and of associated distortion function :

Definition 4.1 (Conversion and distortion functions) Let $f$ any bijective increasing function from $\mathbb{R}$ to $\mathbb{R}$. It is said to be a conversion function. Furthermore the associated distortion function to $f$ is defined by: $T_{f}:[0,1] \rightarrow[0,1]$ such that

$$
T_{f}(u)= \begin{cases}0 & \text { if } u=0, \\ \operatorname{logit}^{-1}(f(\operatorname{logit}(u))) & \text { if } 0<u<1, \\ 1 & \text { if } u=1\end{cases}
$$

Remark that $T_{f}$ is a continuous non-decreasing function, such that $T_{f}(0)=0, T_{f}(1)=1$. Furthermore we remark that the distortions function are chosen in a way to be easily invertible. In particular in a way such that $T_{f} \circ T_{g}=T_{f \circ g}, T_{f}^{-1}=T_{f-1}$. These readily invertible distortions help simulation of the distorted distributions (see Bienvenüe and Rullière, 2012). 


\subsection{Hyperbolic conversion functions}

In this section we consider this particular class of conversion function, which correspond to functions that are defined in Bienvenüe and Rullière (2012).

Definition 4.2 (A class of hyperbole) The considered hyperbole $H$ is

$$
H_{m, h, \rho_{1}, \rho_{2}, \eta}(x)=m-h+\left(e^{\rho_{1}}+e^{\rho_{2}}\right) \frac{x-m-h}{2}-\left(e^{\rho_{1}}-e^{\rho_{2}}\right) \sqrt{\left(\frac{x-m-h}{2}\right)^{2}+e^{\eta-\frac{\rho_{1}+\rho_{2}}{2}} .}
$$

with $m, h, \rho_{1}, \rho_{2} \in \mathbb{R}$, and one smoothing parameter $\eta \in \mathbb{R}$.

After some calculations, one can check that

$$
H_{m, h, \rho_{1}, \rho_{2}, \eta}^{-1}(x)=H_{m,-h,-\rho_{1},-\rho_{2}, \eta}(x) .
$$

In the following we illustrate with some examples the hyperbolic conversion functions proposed in Definition 4.2 .

Example 2 (Global distortion: a bivariate case) We provide an illustration of a bivariate global distorted distribution $\tilde{F}(x, y)$ (see Definition 2.3). As internal distortion we consider the hyperbolic conversion functions proposed in Definition 4.2 with $m=0.536, h=0, \rho_{1}=-\rho_{2}=0.321, \eta=5$.

Let now $C(u, v)=u v$ and $F_{1}(x)=F_{2}(x)=1-\mathrm{e}^{-x}$. Then $F(x, y)=\left(1-\mathrm{e}^{-x}\right)\left(1-\mathrm{e}^{-y}\right)$. Using Definition 2.3, we consider the bivariate distorted distribution function :

$$
\tilde{F}(x, y)=T \circ C\left(T^{-1} F_{1}(x), T^{-1} F_{2}(y)\right),
$$

where $T=T_{f}$, with $f=H_{m, h, \rho_{1}, \rho_{2}, \eta}$. We denote $\tilde{f}$ the associated density function to $\tilde{F}$. For graphical illustration see Figure 2.
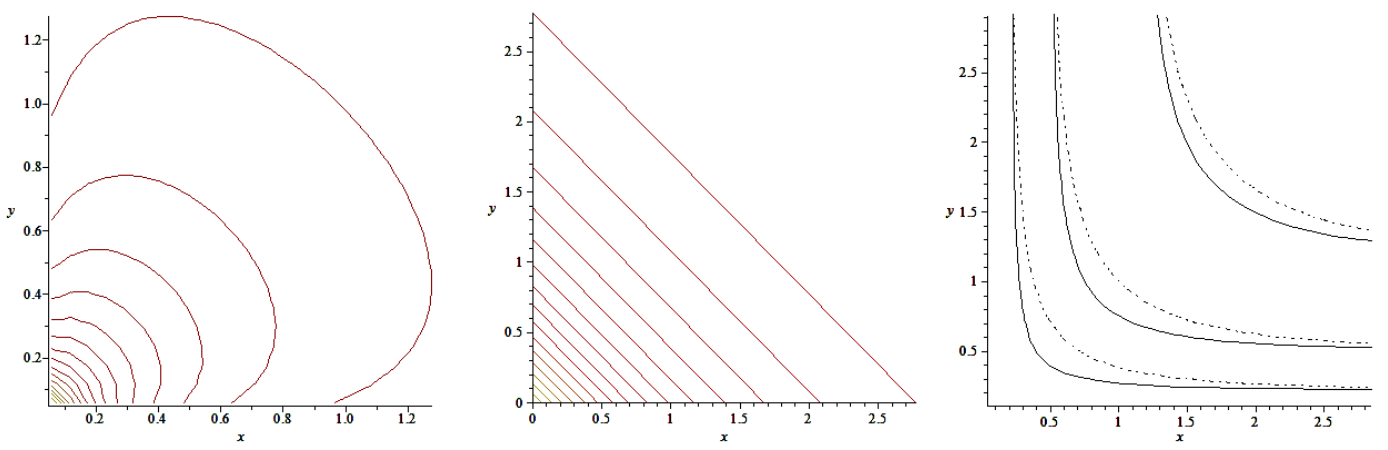

Figure 2: (Left) level curves of distorted density $\widetilde{f}(x, y)$; (Center) level curves of non-distorted independent density $f(x, y)=$ $\mathrm{e}^{-x} \mathrm{e}^{-y}$; (Right) level curves for $\alpha=0.2,0.4,0.7$ of the distorted distribution $\widetilde{F}(x, y)$ (full curves) and the non-distorted independent distribution (i.e., $F(x, y)=\left(1-\mathrm{e}^{-x}\right)\left(1-\mathrm{e}^{-y}\right)$ ) (dashed curves). Parameters: $m=0.536, h=0, \rho_{1}=-\rho_{2}=$ $0.321, \eta=5$.

In the following we illustrate a global distortion in the case of a 3-dimensional distribution function.

Example 3 (Global distortion: a 3-dimensional case) We provide an illustration of a 3-dimensional global distorted distribution $\tilde{F}(x, y, z)$ (see Definition 2.3). We follow the same approach to the bivariate example above. As internal distortion we consider the hyperbolic conversion functions proposed in Definition 4.2 with $m=0.5, h=0, \rho_{1}=-\rho_{2}=0.91, \eta=3$. We remark that with this choice of parameters we 
deal with an absolutely monotonic external distortion, of order 3. Using Definition 2.3, we consider the 3-dimensional distorted distribution function :

$$
\tilde{F}(x, y, z)=T \circ C\left(T^{-1} F_{1}(x), T^{-1} F_{2}(y), T^{-1} F_{3}(z)\right),
$$

where $T=T_{f}$, with $f=H_{m, h, \rho_{1}, \rho_{2}, \eta}$. For graphical illustration see Figure 3 .
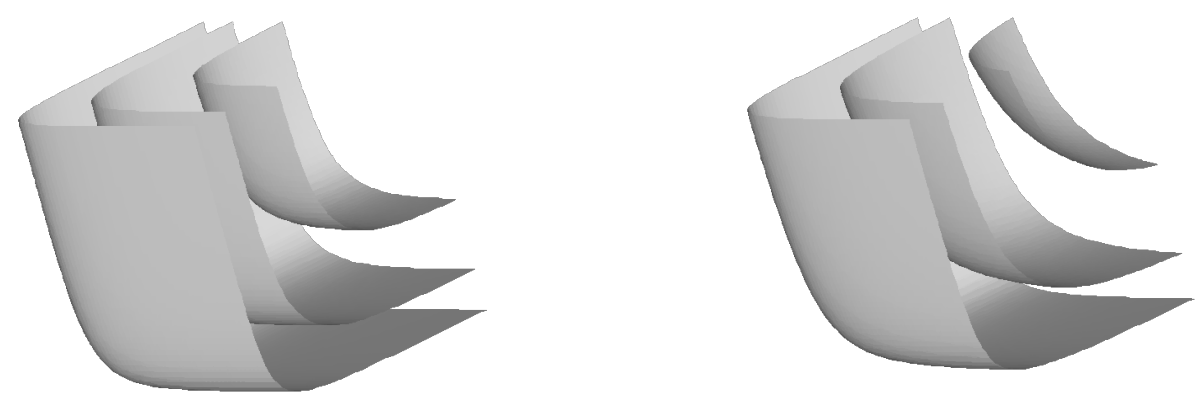

Figure 3: (Left) level curves for $\alpha=0.1,0.4,0.7$ of the 3-dimensional distorted distribution $\widetilde{F}(x, y, z)$, for parameters $m=$ $0.5, h=0, \rho_{1}=-\rho_{2}=0.91, \eta=3$. (Right) level curves for $\alpha=0.1,0.4,0.7$ of the 3-dimensional non-distorted independent distribution with exponential marginals with parameter 1 .

\subsection{Smooth estimation algorithm}

We consider the generic hyperbolic conversion function defined in Equation (13). First remark that when the smoothing parameter $\eta$ tends to $-\infty$, the hyperbole $H$ tends to the angle function:

$$
A_{m, h, \rho_{1}, \rho_{2}}(x)=m-h+(x-m-h)\left(e^{\rho_{1}} 1_{\{x<m+h\}}+e^{\rho_{2}} 1_{\{x>m+h\}}\right) .
$$

As remarked in Bienvenüe and Rullière (2012), it thus appears that hyperbolic distortions have the advantage of being smooth versions of angle functions. They show in their paper that initial parameters for the estimation are easy to obtain with angle compositions.

Definition 4.3 (Composite distortions) Let $k \in \mathbb{N}$. Consider $\eta \in \mathbb{R}$ and a given parameter vector $\theta=\left(m, h, \rho_{1}, \rho_{2}, a_{1}, r_{1}, \ldots, a_{k}, r_{k}\right)$ if $k \geq 1$, or $\theta=\left(m, h, \rho_{1}, \rho_{2}\right)$ if $k=0$. We define the angle composite distortion $\mathcal{A}_{\theta}$ as:

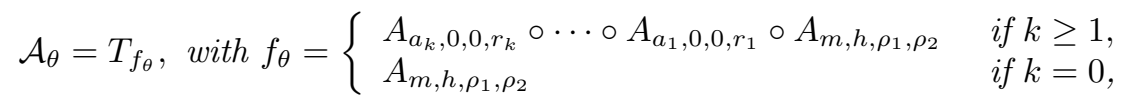

and the hyperbolic composite distortion $\mathcal{H}_{\theta, \eta}$ as:

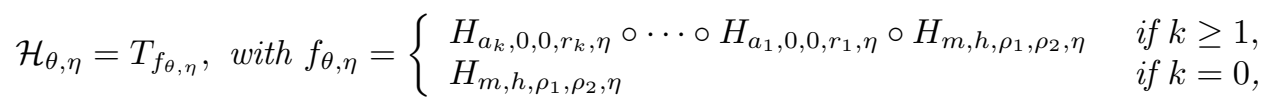

where $A_{m, h, \rho_{1}, \rho_{2}}$ is given in Equation (14), and where $H_{m, h, \rho_{1}, \rho_{2}, \eta}$ is defined in Definition 4.2.

Remark 6 (Inverse composite distortions) Let $k \in \mathbb{N}$. Consider $\eta \in \mathbb{R}$ and a given parameter vector $\theta=\left(m, h, \rho_{1}, \rho_{2}, a_{1}, r_{1}, \ldots, a_{k}, r_{k}\right)$ if $k \geq 1$, or $\theta=\left(m, h, \rho_{1}, \rho_{2}\right)$ if $k=0$. Since $T_{f}^{-1}=T_{f^{-1}}$, the angle composite distortion $\mathcal{A}_{\theta}^{-1}$ is such that:

$$
\mathcal{A}_{\theta}^{-1}=T_{f_{\theta}}, \text { with } f_{\theta}= \begin{cases}A_{m,-h,-\rho_{1},-\rho_{2}} \circ A_{a_{1}, 0,0,-r_{1}} \circ \cdots \circ A_{a_{k}, 0,0,-r_{k}} & \text { if } k \geq 1 \\ A_{m,-h,-\rho_{1},-\rho_{2}} & \text { if } k=0 .\end{cases}
$$


The hyperbolic inverse composite distortion $\mathcal{H}_{\theta, \eta}$ is such that:

$$
\mathcal{H}_{\theta, \eta}^{-1}=T_{f_{\theta, \eta},} \text { with } f_{\theta, \eta}= \begin{cases}H_{m,-h,-\rho_{1},-\rho_{2}, \eta} \circ H_{a_{1}, 0,0,-r_{1}, \eta} \circ \cdots \circ H_{a_{k}, 0,0,-r_{k}, \eta} & \text { if } k \geq 1, \\ H_{m,-h,-\rho_{1},-\rho_{2}, \eta} & \text { if } k=0,\end{cases}
$$

Definition 4.4 (Suited parameters from $\Omega$ ) Let $k \in \mathbb{N}$. Consider one given set $\Omega=\left\{\omega_{1}, \ldots, \omega_{3+k}\right\}$, $\omega_{j} \in(0,1)^{2}$. Denote by $u_{j}$ and $v_{j}$ the two respective components of each $\omega_{j}$ in the logit scale, such that $\omega_{j}=\left(\operatorname{logit}^{-1} u_{j}, \operatorname{logit}^{-1} v_{j}\right), j \in\{1, \ldots, 3+k\}$. Assume that $u_{j}$ and $v_{j}$ are increasing sequences of $j$. We define:

$$
\Theta(\Omega)= \begin{cases}\left(m, h, \rho_{1}, \rho_{2}, a_{1}, r_{1}, \ldots, a_{k}, r_{k}\right) & \text { if } k \geq 1 \\ \left(m, h, \rho_{1}, \rho_{2}\right) & \text { if } k=0\end{cases}
$$

where $m=\frac{u_{2}+v_{2}}{2}, h=\frac{u_{2}-v_{2}}{2}, \rho_{1}=\ln \left(\frac{v_{2}-v_{1}}{u_{2}-u_{1}}\right), \rho_{2}=\ln \left(\frac{v_{3}-v_{2}}{u_{3}-u_{2}}\right), r_{k}=\ln \left(\frac{v_{3+k}-v_{2+k}}{u_{3+k}-u_{2+k}} \frac{u_{2+k}-u_{1+k}}{v_{2+k}-v_{1+k}}\right)$, $a_{k}=v_{2+k}, k \geq 1$.

Proposition 4.1 (Suited composite distortions) Let $k \in \mathbb{N}$. Consider one given set $\Omega=\left\{\omega_{1}, \ldots, \omega_{3+k}\right\}$, $\omega_{j} \in(0,1)^{2}$ and a smoothing parameter $\eta \in \mathbb{R}$. Set $\theta=\Theta(\Omega)$, then

- the distortion $\mathcal{A}_{\theta}(x)$ is piecewise linear in the logit scale and will be called logit-piecewise linear. It links point $(0,0)$, points of $\Omega$, and point $(1,1)$, so that it fulfils conditions of Definition 3.2.

- the distortion $\mathcal{H}_{\theta, \eta}$ converges pointwise to $\mathcal{A}_{\theta}$ as $\eta$ tends to $-\infty$. It results that the continuous and differentiable distortion $\mathcal{H}_{\theta, \eta}$ can fit as precisely as desired the set of points $\Omega$ when $\eta$ tends to $-\infty$.

Proof: The first result is proved in Bienvenüe and Rullière (2012). It simply comes from the fact that $\mathcal{A}_{\Theta(\Omega)}\left(u_{j}\right)=v_{j}$ for all $j \in\{1, \ldots, 3+k\}$, where $\omega_{j}=\left(\operatorname{logit}^{-1} u_{j}\right.$, $\left.\operatorname{logit}^{-1} v_{j}\right)$. The convergence of the hyperbole composite distortion toward the angle composite distortion is straightforward and also evoked in Bienvenüe and Rullière (2012).

Using Algorithm 1, one can find a piecewise or logit-piecewise linear distortion for the external distortion $T$ and corresponding set $\Omega$. Furthermore, using Proposition 2.3, one can build the corresponding internal distortions $T_{1}, \ldots, T_{d}$ and easily propose suited piecewise or logit-piecewise linear internal distortions and corresponding sets $\Omega_{i}$, for $i=1, \ldots, d$.

The corresponding parameters of these sets $\Omega$ and $\Omega_{1}, \ldots, \Omega_{d}$ are given by Definition 4.4. These parameters $\theta=\Theta(\Omega)$ and $\theta_{1}=\Theta\left(\Omega_{1}\right), \ldots, \theta_{d}=\Theta\left(\Omega_{d}\right)$ constitute initial values for distortions of smoothed hyperbolic external and internal distortions. Choosing a convenient smoothing parameter $\eta$, one can define a complete vector parameter:

$$
\vec{\Theta}=\left(\theta_{1}, \ldots, \theta_{d}, \theta, \eta\right)
$$

As seen previously, from now, the estimation of $\vec{\Theta}$ relies exclusively on univariate optimizations.

From estimated vector $\vec{\Theta}$, the corresponding estimated distribution is:

$$
\tilde{F}_{\vec{\Theta}}\left(x_{1}, \ldots, x_{d}\right)=\mathcal{H}_{\theta, \eta} \circ C\left(\mathcal{H}_{\theta_{1}, \eta}^{-1} \circ F_{1}\left(x_{1}\right), \ldots, \mathcal{H}_{\theta_{d}, \eta}^{-1} \circ F_{d}\left(x_{d}\right)\right) .
$$

and the parametric $\alpha$ level-curves are given by Proposition 2.4

$$
\partial \tilde{L}_{\vec{\Theta}}(\alpha)=\left\{\left(F_{1}^{-1} \circ \mathcal{H}_{\theta_{1}, \eta}\left(u_{1}\right), \ldots, F_{d}^{-1} \circ \mathcal{H}_{\theta_{1}, \eta}\left(u_{d}\right)\right),\left(u_{1}, \ldots, u_{d}\right) \in(0,1)^{d}, C\left(u_{1}, \ldots, u_{d}\right)=\mathcal{H}_{\theta, \eta}^{-1}(\alpha)\right\} .
$$


Since initial parameters of $\vec{\Theta}$ are usually close to optimal values, all these parameters can be improved using standard local optimization algorithm, like gradient descent methods:

$$
\vec{\Theta}^{*}=\operatorname{argmax}_{\vec{\Theta}} \ln \mathcal{L}(\vec{\Theta})
$$

with $\mathcal{L}$ the likelihood on the considered data.

Remark 7 Since these initial values are usually close to optimal values, the numerical optimization easily converges. It is important to notice that this optimization could be done directly in theory. However in practice, starting from piecewise linear distortions is necessary to ensure that the optimization converges.

In the following we detail the algorithm-procedure to get smoothed version of distortions obtained in Algorithm 1.

Algorithm 2 Smooth estimation algorithm

\section{Piecewise linear external distortion}

Let $0<\beta_{1}<\ldots<\beta_{m}<1$ be a set of levels,

Obtain distortion levels $\Omega$ from Algorithm 1,

Obtain suited parameters $\theta=\Theta(\Omega)$ from Definition 4.4 and parametric distortion $T=$ $\mathcal{A}_{\theta}$.

\section{Piecewise linear internal distortions}

\section{for i varying from 1 to $d$}

Let $0<\alpha_{1}^{(i)}<\ldots<\alpha_{m_{i}}^{(i)}<1$ be a set of levels associated to the $i$ th-marginal $F_{i}$, Obtain distortion levels $\Omega_{i}=\left\{\left(\alpha_{j}^{(i)}, T_{i}\left(\alpha_{j}^{(i)}\right)\right)\right\}_{j=1, \ldots, m_{i}}$, from Equation (7),

Obtain suited parameters $\theta_{i}=\Theta\left(\Omega_{i}\right)$ from Definition 4.4 and distortions $T_{i}=\mathcal{A}_{\theta_{i}}$. end for

\section{Smoothed distortions}

Choose a smoothing parameter $\eta$,

Improve all parameters including $\eta$ by Equation (17),

Check validity condition $T \in \mathcal{T}$,

Get parametric expression for $\tilde{F}$ and $\partial \tilde{L}$ by Equations (15) and (16).

Remark 8 In Algorithm 2, using the hyperbolic parameterizations in Proposition 4.1, we can fit distortions $T_{1}, \ldots, T_{d}$ as precisely as desired. For the convergence property of these parameterizations, we refer the interested reader to Theorem 2 in Bienvenüe and Rullière (2012). As remarked before these distortions can modify the tail structure of marginal distributions. Then the considered distortions functions are able to adjust also the poor initial choices of marginal distributions.

\subsection{Refinements}

In some particular numerical cases, the choice of the input values of the algorithms may be difficult to do. This section presents some propositions to ease this choice. 
Choice of initial levels. Concerning the choice of initial levels $\beta_{j}$, a problem is that if $T$ is very concave or convex, then levels $\alpha_{j}=T^{-1}\left(\beta_{j}\right)$ may be concentrated on a small interval of $(0,1)$, e.g. $(0.90,1)$, even for values of $\beta_{j}$ spread quite uniformly on $(0,1)$.

Proposed solution: One can modify levels $\beta_{j}$ in order to ensure that both $\alpha_{j}$ and $\beta_{j}$ are reasonably spread over the whole interval $(0,1)$. Let $\Delta$ be the diagonal of equation $y=x$. Assuming that the projection of $\left(\alpha_{j}, \beta_{j}\right)$ on $\Delta$ is $\left(\delta_{j}, \delta_{j}\right)$, one gets $\beta_{j}=-\alpha_{j}+2 \delta_{j}$. Given a distortion $T$ and a set of levels $0<\delta_{1}<\ldots<\delta_{m}<1$, e.g. $\delta_{j}=j /(m+1)$, one can define

$$
\left\{\begin{array}{l}
\alpha_{j}(T)=a \text { such that } T(a)=-a+2 \delta_{j} \\
\beta_{j}(T)=-\alpha_{j}(T)+2 \delta_{j}
\end{array}\right.
$$

Replacing initial levels $\beta_{j}$ by $\beta_{j}\left(T^{\nu}\right)$ at the beginning of each step $\nu$ in Algorithm 1 allows to get a better distribution of elements of the final set $\Omega$ into $(0,1)^{2}$. Similar considerations can be applied to each internal distortion $T_{i}$ : initial levels $\alpha_{j}^{(i)}$ can be replaced by $\alpha_{j}\left(T_{i}\right)$ in Algorithm 2 , for $j=1, \ldots, m_{i}$.

Choice of initial smoothing parameter. Consider a given angle function $A_{m, h, \rho_{1}, \rho_{2}}$ with apex in $(m+$ $h, m-h)$. Replacing this angle by a smooth hyperbole $H_{m, h, \rho_{1}, \rho_{2}, \eta}$ causes some problems. First, the hyperbole is not passing trough the point $(m+h, m-h)$ as the angle function. Second, when $\eta$ is large, the hyperbole gets far from the angle function. In summary, the angle is set to pass through a well chosen point $(m+h, m-h)$, but the hyperbole gets far from this point when smoothing parameter $\eta$ increases. In numerical illustrations, the optimization of parameter $\eta$ thus leads to small values of this parameter, high absolute derivatives of distribution functions at some points, and an insufficient smoothing of the final distortions.

Proposed solution: Define a new hyperbole $H^{*}$ as

$$
\begin{gathered}
H_{m, h, \rho_{1}, \rho_{2}, \eta}^{*}=H_{m, h+\delta\left(\rho_{1}, \rho_{2}, \eta\right), \rho_{1}, \rho_{2}, \eta} \\
\text { with } \delta\left(\rho_{1}, \rho_{2}, \eta\right)=\frac{\mathrm{e}^{\rho_{2}}-\mathrm{e}^{\rho_{1}}}{\sqrt{\left(1+\mathrm{e}^{\rho_{1}}\right)\left(1+\mathrm{e}^{\left.\rho_{2}\right)}\right.}} \mathrm{e}^{-\frac{\rho_{1}+\rho_{2}}{4}} \mathrm{e}^{\eta / 2} .
\end{gathered}
$$

One can check after some calculations that:

- $H_{m, h, \rho_{1}, \rho_{2}, \eta}(m+h)=m-h$, so that the new hyperbole $H^{*}$ is passing trough the point $(m+h, m-h)$.

$$
\text { - } \delta\left(-\rho_{1},-\rho_{2}, \eta\right)=-\delta\left(\rho_{1}, \rho_{2}, \eta\right) \text {, so that } H_{m, h, \rho_{1}, \rho_{2}, \eta}^{*-1}=H_{m,-h,-\rho_{1},-\rho_{2}, \eta}^{*} .
$$

Given a smoothing parameter $\eta$, the proposed improvement is to choose $H_{m, h, \rho_{1}, \rho_{2}, \eta}^{*}$ as a smooth version of the angle $A_{m, h, \rho_{1}, \rho_{2}}$, instead of $H_{m, h, \rho_{1}, \rho_{2}, \eta}$. Corresponding distortion composition will be denoted $\mathcal{H}^{*}$ instead of $\mathcal{H}$. Using $\mathcal{H}^{*}$ instead of $\mathcal{H}$, an univariate maximization of the log-likelihood on the data leads to a good choice of the parameter $\eta$.

Choice of interpolation function $\bar{T}_{\Omega}$ : Piecewise linear distortion $\bar{T}_{\Omega}$ is used in Algorithm 1. It gives interpolations of $T$, when $T$ is passing through all points of $\Omega$. The most simple solution is to choose a simple linear interpolation of points of $\Omega$. The problem is that when $T$ is very concave or convex, a simple linear interpolation may be quite far from the real value of $T$.

Proposed solution: The proposed solution consists in replacing $\bar{T}_{\Omega}$ by $\mathcal{A}_{\Theta(\Omega)}$, or eventually by a smoothed version $\mathcal{H}_{\Theta(\Omega), \eta}^{*}$ for a given parameter $\eta$. 


\section{Numerical applications}

\subsection{A simple bivariate model}

In the following numerical illustrations we consider a very basic independent exponential model where:

$$
F\left(x_{1}, x_{2}\right)=F_{1}\left(x_{1}\right) F_{2}\left(x_{2}\right),
$$

with $F_{1}(x)=F_{2}(x)=1-\mathrm{e}^{-x}, x \in \mathbb{R}^{+}$. We aim at distorting this basic bivariate distribution in order to fit real data. Once distortions $T, T_{1}, T_{2}$ estimated, we obtain analytical expressions for $\widetilde{F}$ and $\partial \widetilde{L}(\alpha)$ :

$$
\tilde{F}\left(x_{1}, x_{2}\right)=T\left(T_{1}^{-1}\left(1-\mathrm{e}^{-x_{1}}\right) \cdot T_{2}^{-1}\left(1-\mathrm{e}^{-x_{2}}\right)\right), \quad \text { for } x_{1}, x_{2} \geq 0,
$$

and for any $\alpha \in(0,1)$, from Proposition 2.4,

$$
\partial \tilde{L}(\alpha)=\left\{\left(-\log \left(1-T_{1}(u)\right),-\log \left(1-T_{2}\left(\frac{T^{-1}(\alpha)}{u}\right)\right)\right), u \in\left(T^{-1}(\alpha), 1\right)\right\} .
$$

Distorted marginals in this simple bivariate model are given by:

$$
\tilde{F}_{1}(x)=T \circ T_{1}^{-1}\left(1-\mathrm{e}^{-x}\right) \text { and } \tilde{F}_{2}(x)=T \circ T_{2}^{-1}\left(1-\mathrm{e}^{-x}\right),
$$

and corresponding distorted copula is

$$
\tilde{C}(u, v)=T\left(T^{-1}(u) \cdot T^{-1}(v)\right) .
$$

In this independent case the regular condition of Proposition 2.6 becomes:

$$
T^{\prime}(x)+x T^{\prime \prime}(x) \geq 0, \quad \text { for any } x \in[0,1] .
$$

This simple initial independent exponential multivariate distribution is very far from the data that will be considered. It has been chosen to demonstrate the ability of the estimation procedure to fit real data even when starting with a very poor initial distribution and dependence structure.

\subsection{Real case study: Loss-ALAE data}

In this section we present a real case for which we illustrate the behavior of our algorithm, presented in Sections 3 and 4. We consider the uncensored Loss-ALAE data in the logarithmic scale (for details see Frees and Valdez, 1998). The data size is $n=1500$. Each claim consists of an indemnity payment (the loss, $X$ ) and an allocated loss adjustment expense (ALAE, $Y$ ). Examples of ALAE are the fees paid to outside attorneys, experts, and investigators used to defend claims. We now implement Algorithm 1 and 2 on Loss-ALAE data.

External distortion $T$ :

From Algorithm 1 we obtain the piecewise linear external distortion $\bar{T}_{\Omega}(x)$, for $x \in[0,1]$ with the matrix $\Omega=\{(0.0664662,0.1),(0.6316830,0.5),(0.9762340,0.9)\}$. Furthermore, from set $\Omega$, by applying Definition 4.4 , the suited angle composition-distortion is $\mathcal{A}_{\theta}$, with $\theta=\Theta(\Omega)$.

Internal distortions $T_{i}$ :

From Proposition 4.1 we obtain that the estimated marginal distortions $T_{1}$ and $T_{2}$ can be represented using angle compositions $T_{1}=\mathcal{A}_{\theta_{1}}$ and $T_{2}=\mathcal{A}_{\theta_{2}}$ with associated parameters. Remark that $T_{1}^{-1}=\mathcal{A}_{\theta_{1}}^{-1}$ and $T_{2}^{-1}=\mathcal{A}_{\theta_{2}}^{-1}$ can be directly obtained by Remark 6 .

Including smoothing parameters:

We now include smoothing hyperbolic distortions instead of angle compositions, with $H_{m, h, \rho_{1}, \rho_{2}, \eta}$ as in (13) and $\mathcal{H}_{\theta, \eta}$ as in Definition 4.3. In this case we choose a smoothing parameter for the external distortion (i.e. $\eta$ ) and two smoothing parameters the internal ones (i.e. $\eta_{1}$ and $\eta_{2}$ ). Using the global optimization presented in Equation (17) we obtain: 


\begin{tabular}{|c|c|c|c|c|c|}
\hline Parameters & $m$ & $h$ & $\rho_{1}$ & $\rho_{2}$ & $\eta$ \\
\hline$\theta$ & 0.269721 & 0.2697219 & -0.3702292 & -0.3684284 & 0 \\
\hline$\theta_{1}$ & 4.501394 & -4.501394 & -0.4490202 & -0.3883775 & -3 \\
\hline$\theta_{2}$ & 4.172548 & -4.172548 & -0.5253705 & -0.6708913 & -3 \\
\hline
\end{tabular}

In Figure 4 we have drawn the obtained external distortion $\bar{T}_{\Omega}$, its inverse $\bar{T}_{\Omega}^{-1}$ (using matrix $\Omega$ from Algorithm 1) and the smoothed distortion $\mathcal{H}_{\theta, \eta}^{*}$ (using parameters of table above).

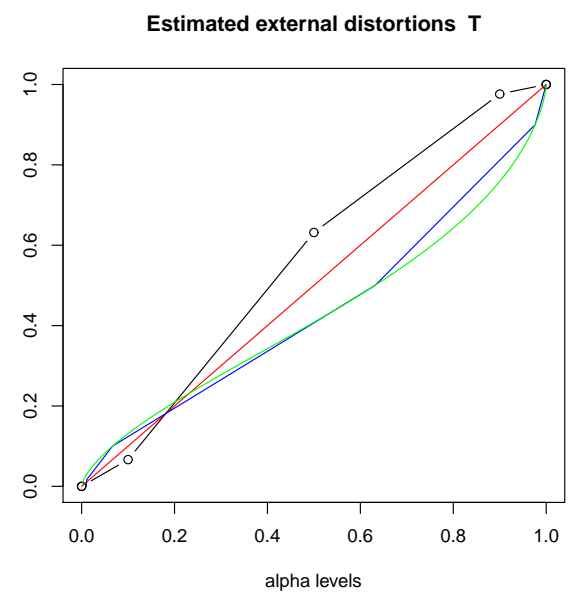

Figure 4: $T(x)=\bar{T}_{\Omega}(x)$ (blue line), $T^{-1}(x)=\bar{T}_{\Omega}^{-1}(x)$ (black line), smoothed $\mathcal{H}_{\theta, \eta}(x)$ (green line), with $\eta=0$. Red line is bisectrix of the first quadrant.

Furthermore, from Proposition 2.3, we get $\tilde{F}_{i}=T \circ T_{i}^{-1} \circ F_{i}$, for $i=1,2$, where $T, T_{i}^{-1}$ are obtained above and $F_{i}$ are the known initial marginals (in this case $F_{1}(x)=F_{2}(x)=1-\mathrm{e}^{-x}$ ). The results are gathered in Figures 5 and 6 below.
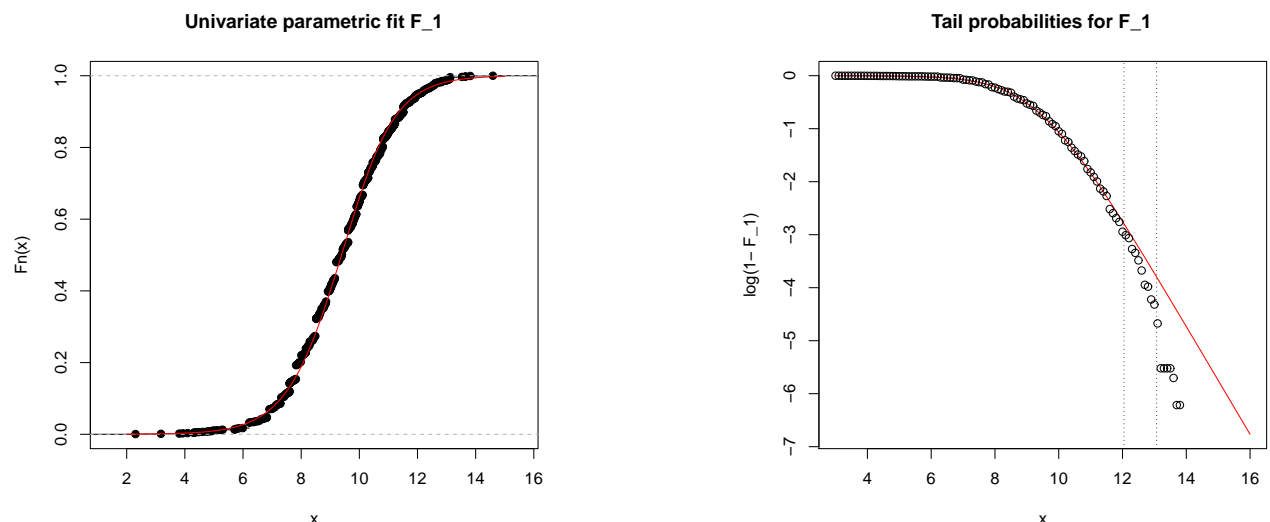

Figure 5: Loss data. (Left) $\tilde{F}_{1}$ (red) and the empirical distribution function of ALAE data (black). (Right) Black points are plotted at empirical tail probabilities calculated from empirical distribution function (in $\log$ scale). Red line is $1-\tilde{F}_{1}(x)$ (in $\log$ scale) for $x \in(3,16)$ (Remark the Loss-ALAE data are transformed in log scale at the beginning of the study). The vertical dotted lines show estimates of $95 \%$ and $99 \%$ VaR (univariate quantile) for the Loss data. 

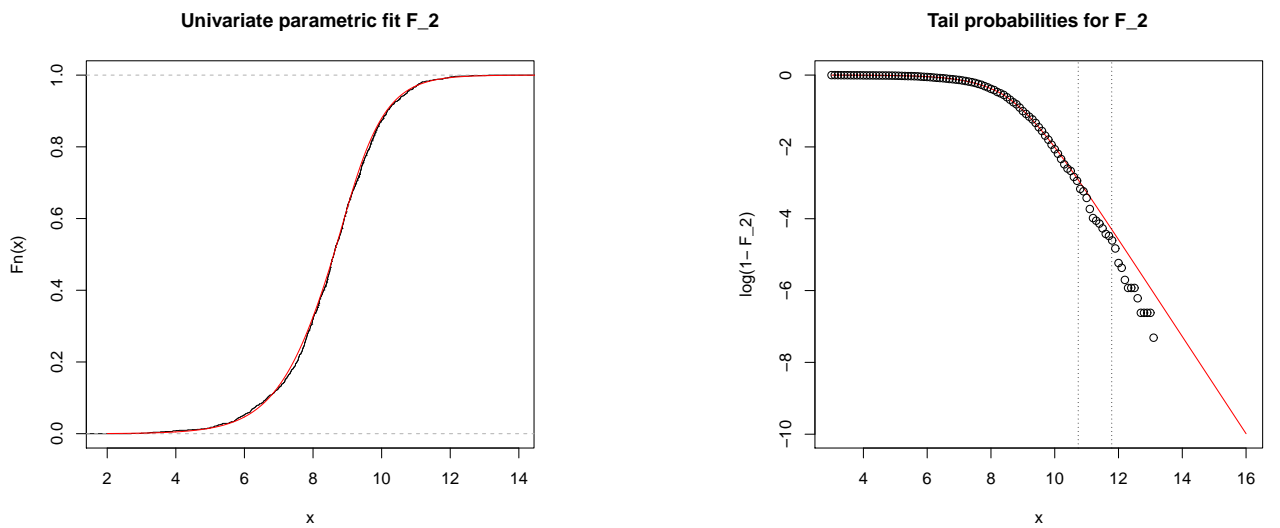

Figure 6: ALAE data. (Left) $\tilde{F}_{2}$ (red) and the empirical distribution function of ALAE data (black). (Right) Black points are plotted at empirical tail probabilities calculated from empirical distribution function (in log scale). Red line is $1-\tilde{F}_{2}(x)$ (in log scale) for $x \in(3,16)$ (Remark the loss-ALAE data are transformed in log scale at the beginning of the study). The vertical dotted lines show estimates of $95 \%$ and $99 \%$ VaR (univariate quantile) for the ALAE data.

Remark 9 Figures 5-6 show the quality of the adjustment for the distorted marginal distributions with respect to the empirical ones. Since in risk assessment we are interested to consider large values of risk $\alpha$ (for instance $\alpha \in(0.95,0.99)$ ), Figures 5-6(right) amplify the tail region using the log-scale (e.g., for the same procedure see Section 7.2.3 in McNeil et al., 2005). The vertical dotted lines show estimates of 95\% and 99\% VaR (univariate quantile) for the marginal distributions. We remark that the estimation procedure presented in this paper is not adapted to optimally fit tail regions in the considered multivariate distribution. In particular, in Algorithm 1 we use classical empirical methods in order to estimate the proximity index (PI). It could be interesting to adapt this work in order to estimate the multivariate quantile for large value of $\alpha$, using suitable tail estimations from the recent extreme theory literature. For instance, a possible starting point, it could be to fix the asymptotic behavior for the marginal distortions, using the regularly varying properties of the distribution tails. The deep investigation of these aspects are potential improvements to this paper that will be investigated in a future work. However, in Section 5.4, we illustrate how our procedure can be improved in the tails by a suitable choice of the levels $\alpha_{i}$ in the matrix $\Omega$ (see Algorithm 1).

We check that the smoothed distortion $T$ satisfies the regular condition of Equation (19) on all points of $(0,1)$. For a graphical representation of the smoothed distortion $T$, i.e. $\mathcal{H}_{\theta, \eta}$, with $\eta=0$, see Figure 4 , green line. The log-likelihood of this model on the Loss-ALAE data is -32147.81 and the associated Akaike Information Criterion is 64325.62 .

In the literature the fit of Loss-Alae data has received some attention. For instance Frees and Valdez (1998) fit on these data a Gumbel-Hougaard copula with parameter $\theta=1.453$; Loss $\sim$ Pareto with parameter $(14.036,1.122)$ and ALAE $\sim$ Pareto with parameter $(14.219,2.118)$. The obtained log-likelihood on the Loss-Alae data of the Frees and Valdez's model is -49075.82 , and the associated AIC is 98161.64. Furthermore in Klugman and Parsa (1999), they propose a fitted model on Loss-Alae data such that the log-likelihood is -31767.9 and the associated AIC is 63545.8 . We remark that these results belong to the same order of magnitude. Our procedure can be compared with these methods and used to obtain the same type of performance. However, differently with respect to the Frees and Valdez's model or the Klugman and Parsa's model, we do not need to assume that our distribution belongs to a particular class of model. More precisely, Klugman and Parsa (1999) checked for instance the maximum likelihood estimation in a class of 15 models. In this sense our procedure can be applied in a more general case, also without choosing preliminarily a fixed class of models to estimate. 
In Figure 7, we compare our method with the empirical level curves defined by $\left\{(x, y) \in \mathbb{R}_{+}^{2}: F_{n}(x, y)=\alpha\right\}$, where $F_{n}$ is the bivariate empirical estimator of $F$, for $\alpha=0.2,0.5,0.8$. We have drawn the parametric $\alpha$ level-curves, given by Proposition 2.4, $\partial \tilde{L}_{\vec{\Theta}}(\alpha)$ (see Equation (16)), using smoothed hyperbolic external and internal distortions (as described by Algorithm 2).

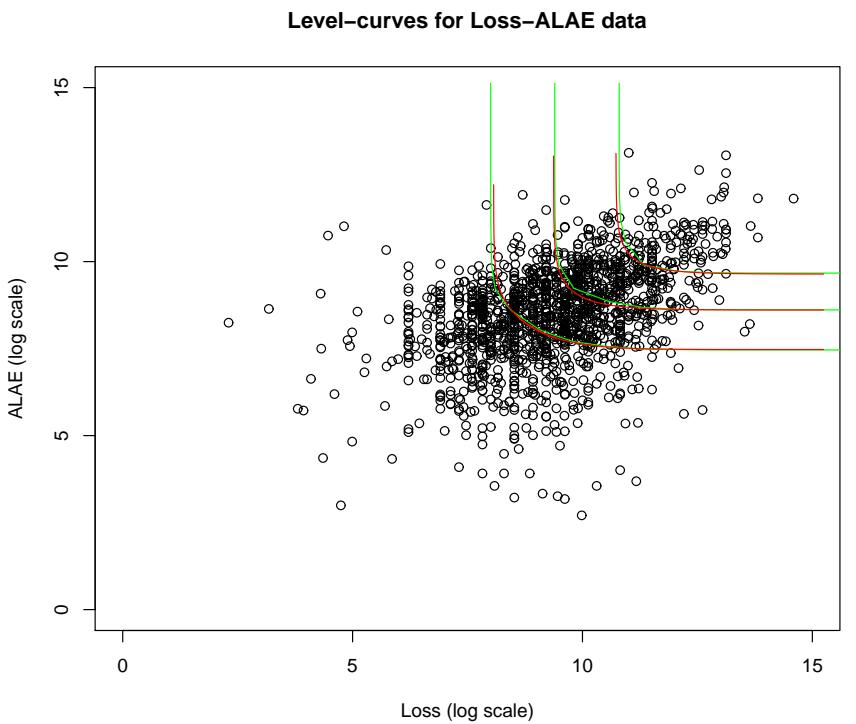

Figure 7: ALAE versus Loss data (in logarithmic scale). Estimated smoothed $\alpha$ level-curves as in Equation (16) (red curves); Empirical level curves $\left\{(x, y) \in \mathbb{R}_{+}^{2}: F_{n}(x, y)=\alpha\right\}$ (green curves); for $\alpha=0.2,0.5,0.8$.

Note that the smoothing parameter $\eta$ is not really crucial with respect to the quality of our estimation, even if it improves the estimation of the level-curves. However it is important in order to obtain differentiable representations of level curves $\partial \widetilde{L}(\alpha)$. In Figure 8(left) we draw the final distorted distribution $\tilde{F}\left(x_{1}, x_{2}\right)$ and its level-curves; in Figure 8(right) we present the level-curves of distorted density function $\tilde{f}\left(x_{1}, x_{2}\right)$. In particular Figure 8(right) illustrates the good quality of the fit of proposed model on the bivariate Loss-ALAE data.
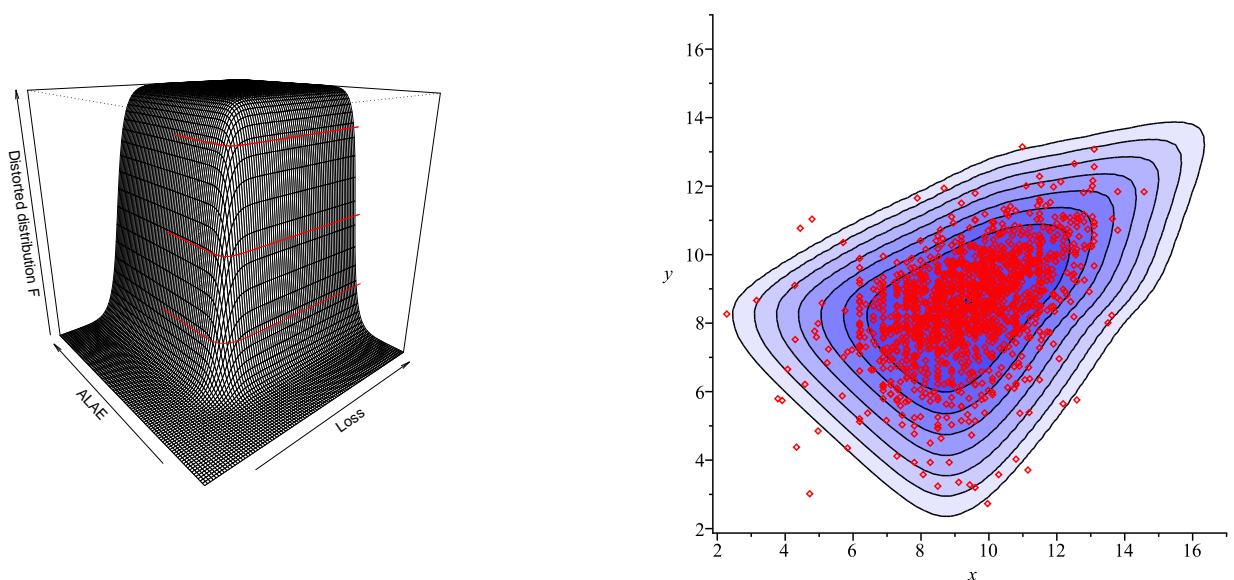

Figure 8: Distorted distribution $\tilde{F}\left(x_{1}, x_{2}\right)$ whit associated distorted level curves (red curves) (left); Level curves of distorted density $\tilde{f}\left(x_{1}, x_{2}\right)$ and Loss-ALAE data (red points) (right). 


\subsection{Real case study: Old Faithful Geyser Data}

In this section we study a second real case that presents an interesting bimodal behavior. This data concern 272 eruptions of the Old Faithful geyser in Yellowstone National Park. Each observation consists of two measurements: the duration (in min) of the eruption $(X)$, and the waiting time (in min) before the next eruption $(Y)$. This waiting time has been divided by 20 in order to get the same order of magnitude on both axis. The interested reader is referred for instance to Obereder et al. (2007), Biernacki et al. (2007). We follow the same estimation procedure presented in Section 5.2 above, for Loss-Alae data.

From Algorithm 1 we obtain the piecewise linear external distortion $\bar{T}_{\Omega}(x)$, for $x \in[0,1]$ and the suited angle composition-distortion with the following vector of parameters:

\begin{tabular}{|c|c|c|c|c|}
\hline Parameters & $m$ & $h$ & $\rho_{1}$ & $\rho_{2}$ \\
\hline$\theta$ & 2.36298 & 2.36298 & -1.2228 & -0.511403 \\
\hline
\end{tabular}

The estimated marginal distortions $T_{1}$ and $T_{2}$ can be represented using angle compositions $T_{1}=\mathcal{A}_{\theta_{1}}$ and $T_{2}=\mathcal{A}_{\theta_{2}}$ with associated parameters:

\begin{tabular}{|c|c|c|c|c|c|c|c|c|c|c|c|c|}
\hline Parameters & $m$ & $h$ & $\rho_{1}$ & $\rho_{2}$ & $a_{1}$ & $r_{1}$ & $a_{2}$ & $r_{2}$ & $a_{3}$ & $r_{3}$ & $a_{4}$ & $r_{4}$ \\
\hline$\theta_{1}$ & 0.142678 & -1.57529 & -3.63924 & -1.75552 & 2.3049 & 2.42343 & 3.28006 & -1.38934 & 3.82849 & -0.767873 & 4.48883 & -0.592127 \\
\hline$\theta_{2}$ & 1.12089 & -1.50953 & -2.93159 & -1.72849 & 2.94893 & 0.659211 & 3.62367 & -0.861415 & 3.87955 & 0.095106 & 4.13411 & -0.323934 \\
\hline
\end{tabular}

As in the Loss-ALAE model (see Section 5.2) we now include the smoothing hyperbolic distortions indeed of angle compositions. For these data, we get: $\eta=-0.9, \eta_{1}=-9, \eta_{2}=-7.5$. The log-likelihood of our final smoothed model on the Old Faithful geyser data is -1156 and the associated Akaike Information Criterion is 2374. In the literature the fit of Old Faithful geyser data has received some attention (see for instance Obereder et al., 2007). Biernacki et al. (2007) fit on these data a Gaussian mixture model. In this case, they obtain a log-likelihood equal to -1124 and a AIC equal to 2260. As remarked in Section 5.2, that these results belong to the same order of magnitude, however our model does not assume previously a particular form for the fitted distribution.

Furthermore, from Proposition 2.3, we get $\tilde{F}_{i}=T \circ T_{i}^{-1} \circ F_{i}$, for $i=1,2$, where $T, T_{i}^{-1}$ are obtained above and $F_{i}$ are the known initial marginals (in this case $F_{1}(x)=F_{2}(x)=1-\mathrm{e}^{-x}$ ). The results are drawn in Figure 9 and 10 below.

In Figure 11 (left) we draw the final distorted distribution $\tilde{F}\left(x_{1}, x_{2}\right)$ and its level-curves. Figure 11 (right) illustrates the good quality of the fit of proposed model in the diagonal distorted distribution $\tilde{F}(x, x)$.

Furthermore we present the level-curves of distorted density function and we illustrate the role of the smoothing parameters $\eta, \eta_{1}$ and $\eta_{2}$. Indeed the choice of these smoothing parameters is important in order to obtain differentiable representations of level curves $\partial \widetilde{L}(\alpha)$. In Figure 12 we draw the level-curves of distorted density function $\tilde{f}\left(x_{1}, x_{2}\right)$ for different choices of $\eta, \eta_{1}$ and $\eta_{2}$.

We also remark how our distortions can be able to capture the bimodal behavior of these data (see Figure 11, right). Despite its observable complexity, the proposed parametric fit also allows to get a relatively simple parametric expression for level curves, using Equation (18) (see Figure 12). 

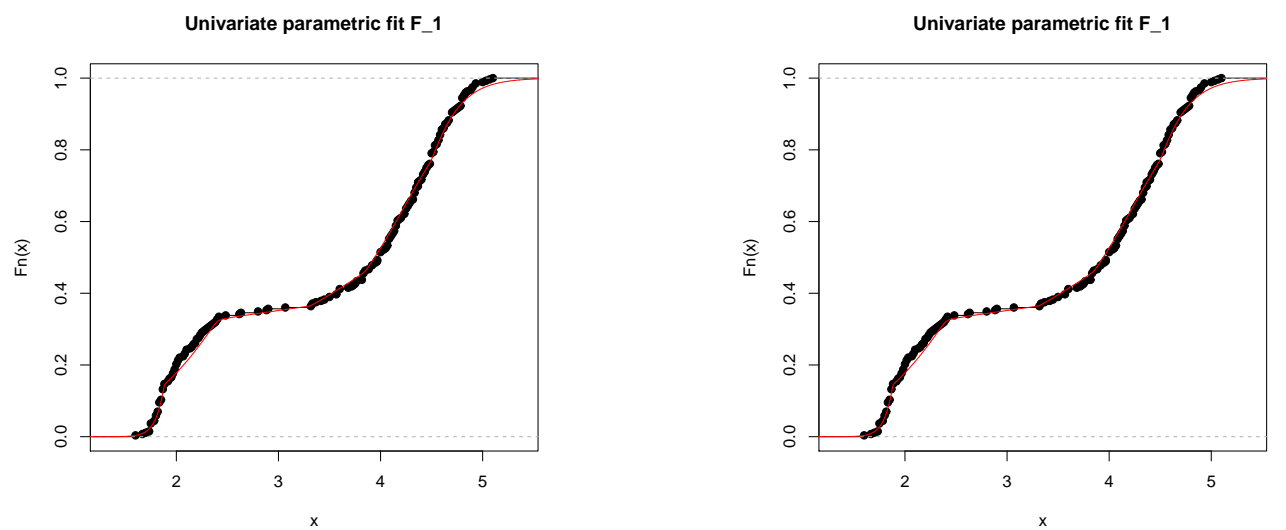

Figure 9: Duration (in min) of the eruption data. (Left) $\tilde{F}_{1}$ (red) and the empirical distribution function of eruption data (black). (Right) Black points are plotted at empirical tail probabilities calculated from empirical distribution function (in $\log$ scale). Red line is $1-\tilde{F}_{1}(x)$ (in log scale). The vertical dotted lines show estimates of $95 \%$ and $99 \%$ VaR (univariate quantile) for the eruption data.
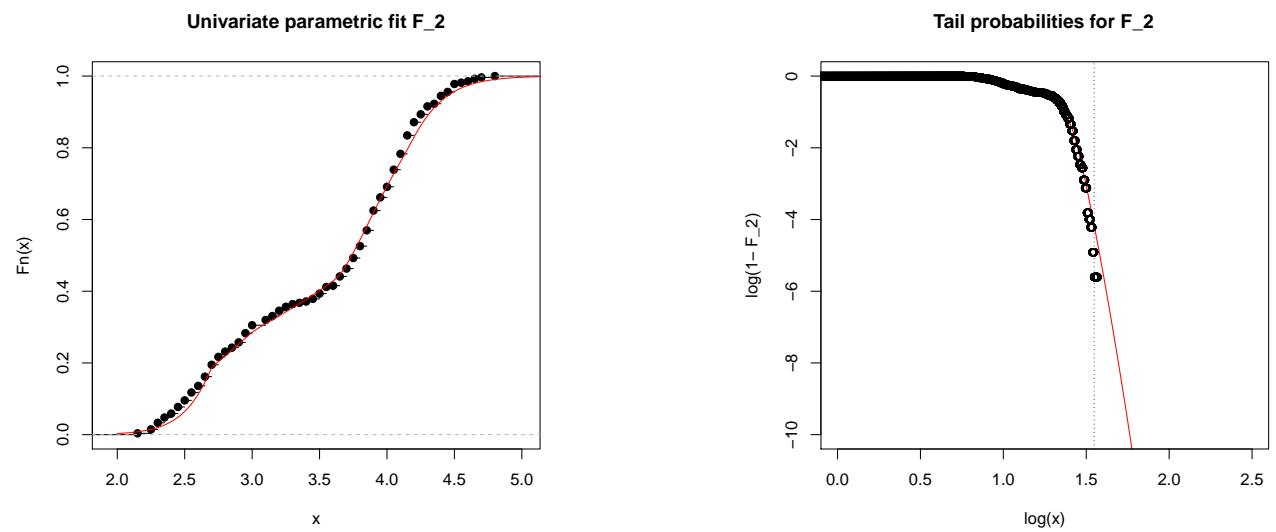

Figure 10: Waiting time (in min) before the next eruption data. (Left) $\tilde{F}_{2}$ (red) and the empirical distribution function of waiting time data (black). (Right) Black points are plotted at empirical tail probabilities calculated from empirical distribution function (in $\log$ scale). Red line is $1-\tilde{F}_{2}(x)$ (in log scale) for the waiting time data.

\subsection{Simulation study}

In a simulated environment we analyse the impact of the choice of levels $\alpha_{i}$ in our procedure (Algorithm 1). In particular we focus the estimation in the tail regions. We illustrate in this section, how our fitting procedures in extreme risk areas (i.e., $\widetilde{F}\left(x_{1}, x_{2}\right)$ for large values of $x_{1}$ and $x_{2}$ ) can be improved by suitable choices of levels in Algorithm 1.

We consider a bivariate sample $\left\{\left(X_{1}^{(i)}, X_{2}^{(i)}\right)\right\}_{i=1, \ldots, n}$, for $n=7000$. Let $\left(X_{1}, X_{2}\right)$ a random vector with Clayton copula structure with parameter $\theta=1$ and marginals $X_{1} \sim \operatorname{Exp}(1)$ and $X_{2} \sim \operatorname{Burr}(1,4)$.

Firstly we consider in Algorithm 1 the set of $\alpha_{i}$ levels:

$$
\begin{cases}\{0.24,0.82,0.98\}, & \text { to construct the first marginal distortions }\left(T_{1}\right) ; \\ \{0.15,0.83,0.99\}, & \text { to construct the second marginal distortions }\left(T_{2}\right) ; \\ \{0.25,0.60,0.98\}, & \text { to construct the external one }(T) .\end{cases}
$$



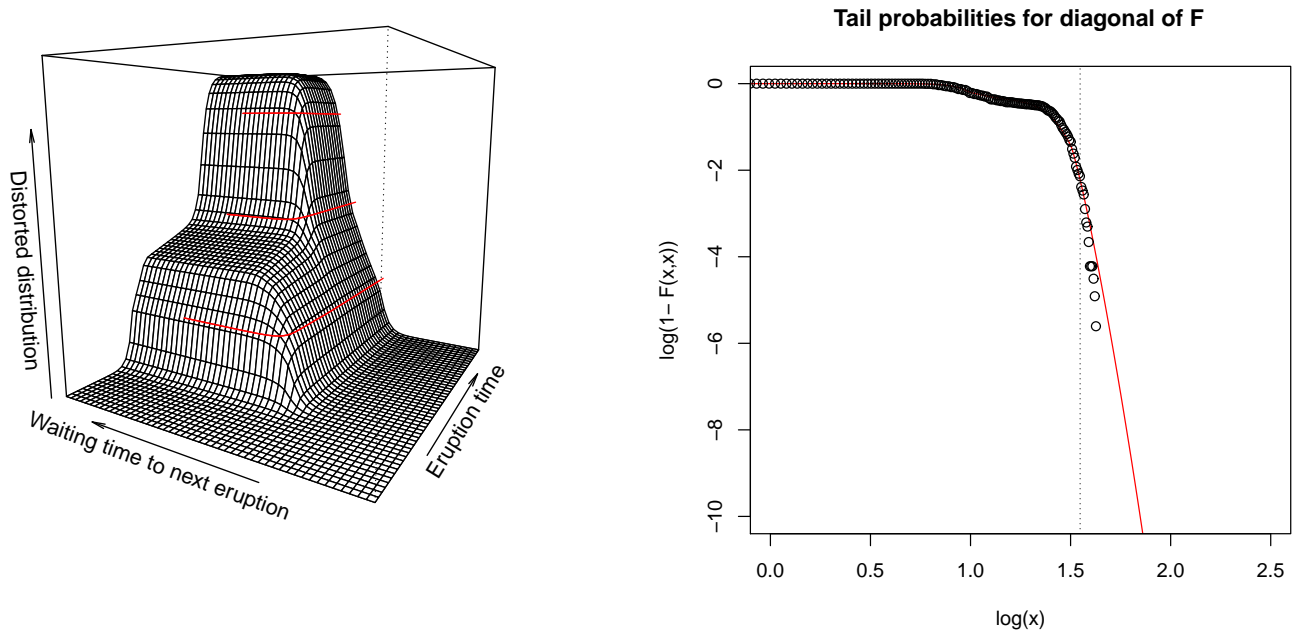

Figure 11: (Left) Distorted distribution $\tilde{F}\left(x_{1}, x_{2}\right)$ whit associated distorted level curves (red curves). (Right) Black points are plotted at empirical tail probabilities on the diagonal, calculated from the empirical bivariate distribution (in log scale). Red line is $1-\tilde{F}(x, x)$ (in log scale). The vertical dotted lines show estimate of $90 \%$ VaR (i.e., the univariate quantile in log scale).
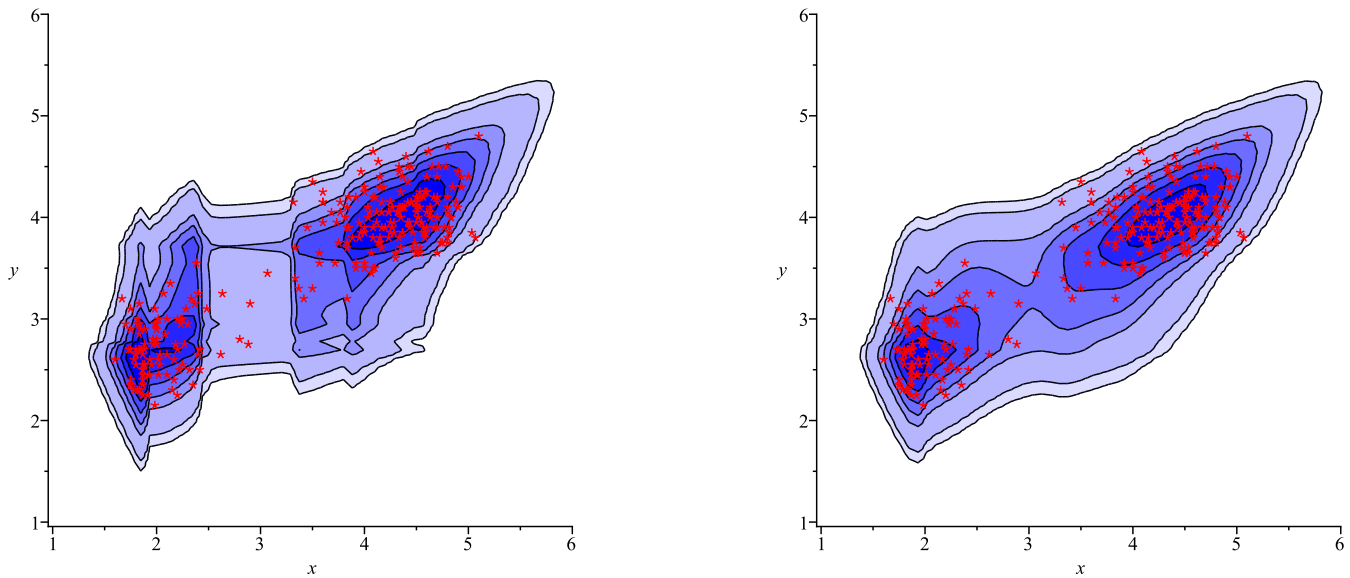

Figure 12: Level curves of distorted density $\tilde{f}\left(x_{1}, x_{2}\right)$ and Old Faithful geyser data (red points: (left) parameter setting $\eta=-0.9, \eta_{1}=-9, \eta_{2}=-7.5 ;$ (right) parameter setting $\eta=-0.9, \eta_{1}=-4, \eta_{2}=-4$.

Then, the obtained log-likelihood on these simulated data is -10611 and the associated Akaike Information Criterion is 21252 .

Figure 13 shows the quality of the adjustment for the distorted marginal distributions (red lines) with respect to the theoretical (green lines) and the empirical (black points) ones. One can see that a choice of $\alpha_{i}$ levels as in (20) provides a good global fit but a non-performing estimation in the tail region of the distribution. This aspect is particularly evident in the case of the heavy-tail marginal distribution $X_{2} \sim$ $\operatorname{Burr}(1,4)$ (see Figure 13, right). We illustrate this phenomenon both for the marginal components (Figure 13 ) and for the diagonal of the bivariate distribution $F(x, x)$ (Figure 16, left). 

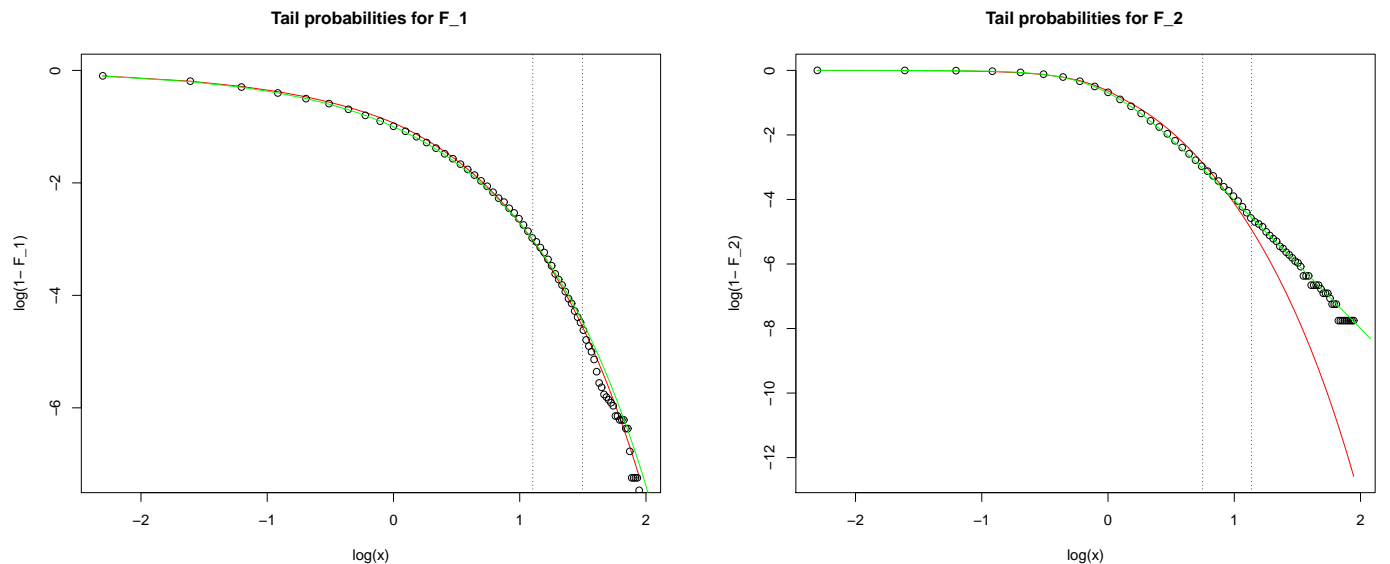

Figure 13: Simulated data: Clayton copula structure with parameter $\theta=1$ and marginals $X_{1} \sim \operatorname{Exp}(1)$ and $X_{2} \sim \operatorname{Burr}(1,4)$. Black points are plotted at empirical tail probabilities calculated from the empirical marginal distributions (in log scale). Red line is $1-\tilde{F}_{1}(x)$ (in log scale) (left) and $1-\tilde{F}_{2}(x)$ (in $\log$ scale) (right) with choice of $\alpha_{i}$ as in (20). The vertical dotted lines show estimates of $95 \%$ and $99 \%$ VaR (i.e., the univariate quantiles in log scale)
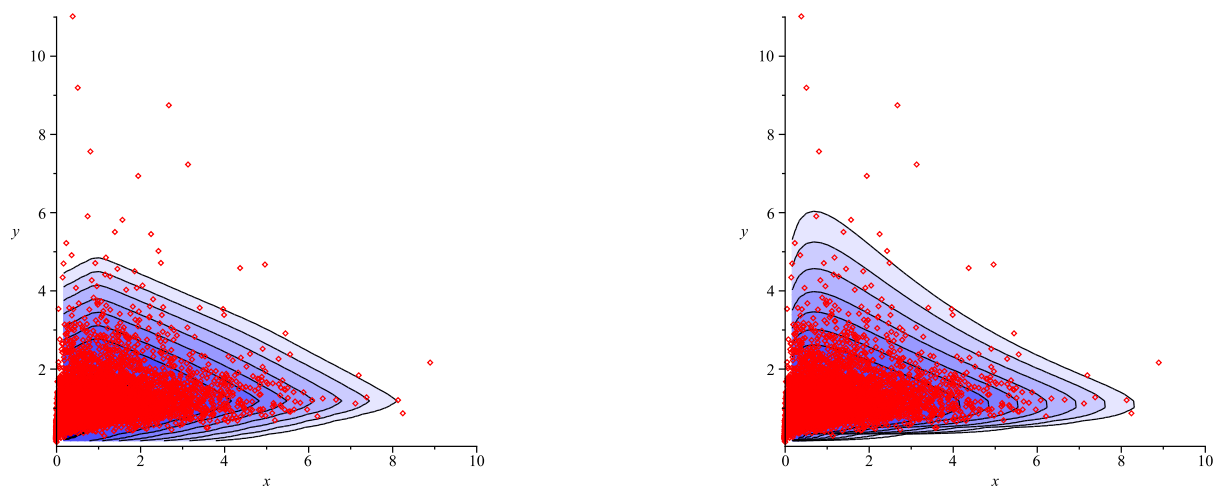

Figure 14: Level curves of distorted density $\tilde{f}\left(x_{1}, x_{2}\right)$ (left) and of the theoretical one (right). We choose $\alpha_{i}$ in Algorithm 1 as in (20). Simulated data: Clayton copula structure with parameter $\theta=1$ and marginals $X_{1} \sim \operatorname{Exp}(1)$ and $X_{2} \sim \operatorname{Burr}(1,4)$ (red points).

As noticed in Remark 9, in risk management we are often interested to consider the risk measure $\operatorname{VaR}_{\alpha}(F)$ (see Equation (1)) of large values of $\alpha$ (e.g., $\alpha \in[0.95,0.99]$ ). In this case we only focus on the multivariate tail regions and we choose large $\alpha_{i}$ levels in the fitting procedure both for the marginals and the copula structure. In particular we take in Algorithm 1 the set of $\alpha_{i}$ levels:

$$
\begin{cases}\{0.95,0.990,0.995\}, & \text { to construct the external distortion } T \\ \{0.95,0.990,0.995\}, & \text { to construct the marginal distortion } T_{1} \\ \{0.95,0.995,0.999\}, & \text { to construct the marginal distortion } T_{2}\end{cases}
$$

Obviously we obtain a degraded global fit: the log-likelihood is -14011.14 and the associated Akaike Information Criterion is 28052.28. Conversely if you only focus on the bivariate tail region (e.g., $x \geq$ $F_{X_{1}}^{-1}(0.95), y \geq F_{X_{2}}^{-1}(0.95)$ ) we obtain satisfactory results both for the marginal components (see Figure 15) and for the diagonal of the bivariate distribution (see Figure 16, right). From the comparison between Figure and Figure 13 (right) and 15 (right), we conclude that this choice of levels $\alpha_{i}$ permits to better fit the heavy-tail marginal distribution $X_{2}$. 
As a summary, centered input levels give a good likelihood whereas extreme levels give good tails. One interest of the proposed distortion is that one can choose both centered and extremal input levels by increasing the number of parameters.
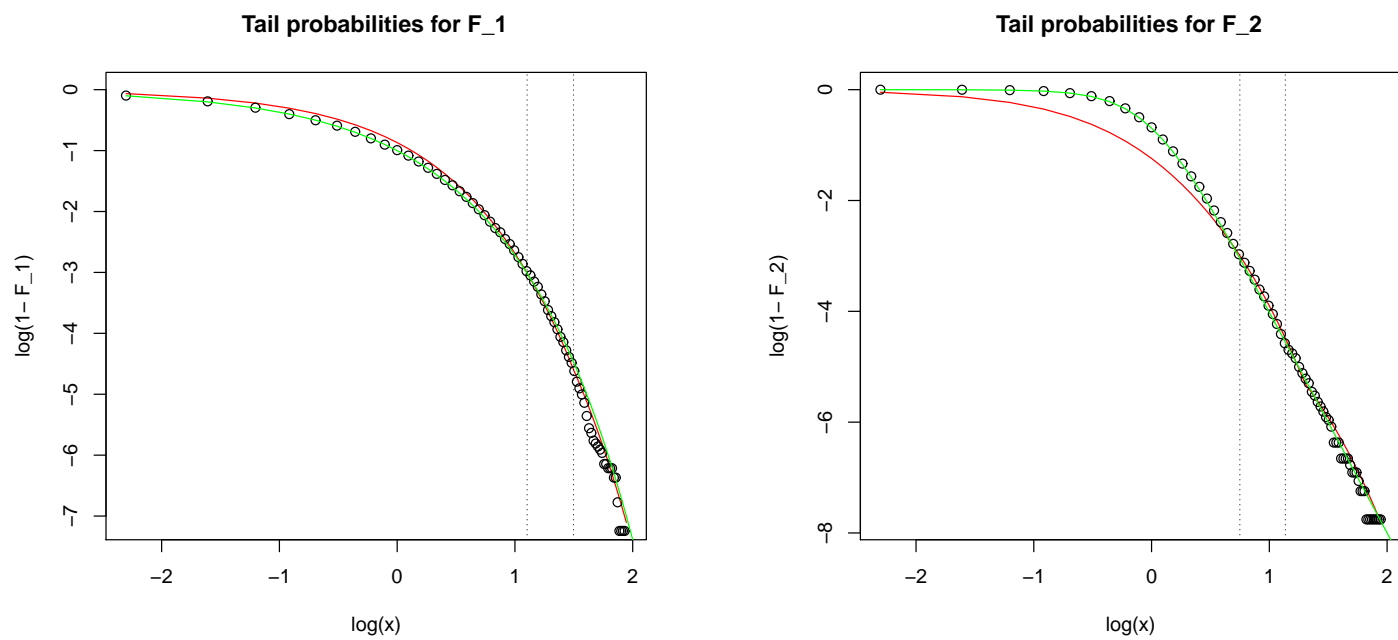

Figure 15: Simulated data: Clayton copula structure with parameter $\theta=1$ and marginals $X_{1} \sim \operatorname{Exp}(1)$ and $X_{2} \sim \operatorname{Burr}(1,4)$. Black points are plotted at empirical tail probabilities calculated from the empirical marginal distributions (in log scale). Red line is $1-\tilde{F}_{1}(x)$ (in $\log$ scale) (left) and $1-\tilde{F}_{2}(x)$ (in $\log$ scale) (right) for the choice of $\alpha_{i}$ as in (21). The vertical dotted lines show estimates of $95 \%$ and $99 \%$ VaR (i.e., the univariate quantiles in log scale).
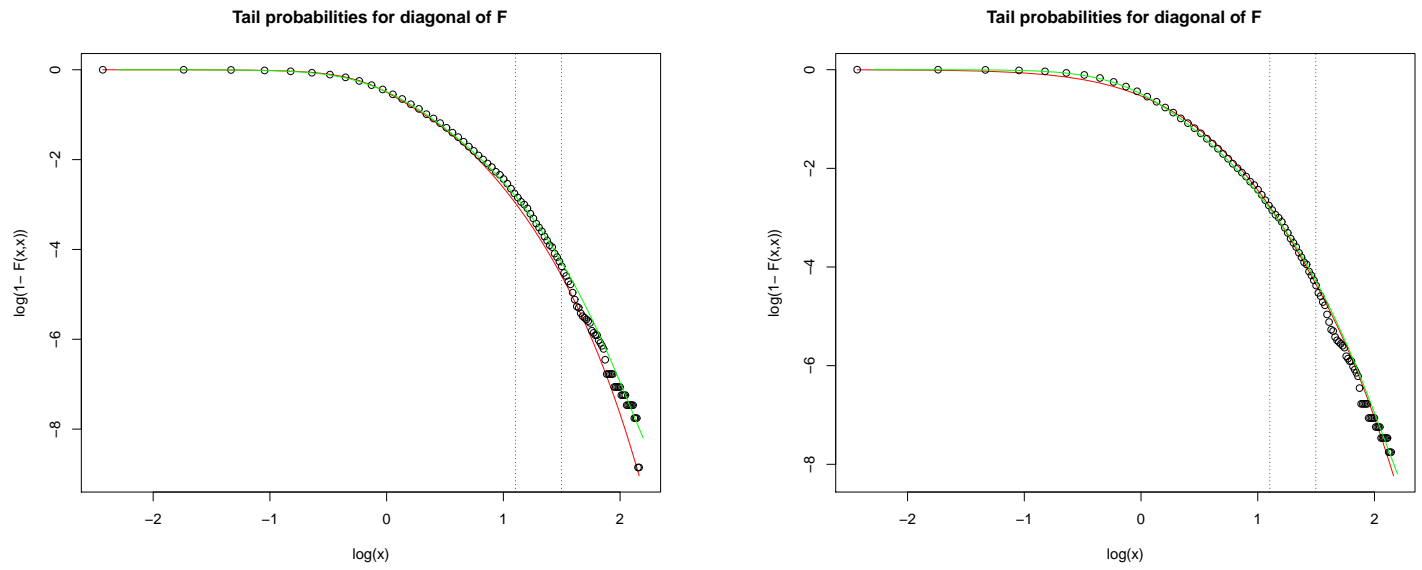

Figure 16: Simulated data: Clayton copula structure with parameter $\theta=1$ and marginals $X_{1} \sim \operatorname{Exp}(1) \operatorname{and} X_{2} \sim \operatorname{Burr}(1,4)$. Black points are plotted at empirical tail probabilities on the diagonal, calculated from the empirical bivariate distribution (in log scale). Red line is $1-\tilde{F}(x, x)$ (in log scale). The fit is obtained with choice of $\alpha_{i}$ as in (20) (left) and as in (21) (right). The vertical dotted lines show estimates of $95 \%$ and $99 \%$ VaR (i.e., the univariate quantiles in log scale)

\section{Conclusion}

Probability distortions allow to build new classes of multivariate distribution functions. Impacts of such distortions on multivariate level curves have been studied. Among interesting properties, we have seen 
that external distortions are only acting on the univariate level of a level curve. Finding the best distorted levels on a particular data directly gives external distortion point values, and only requires univariate optimizations. Following this idea, we have proposed two algorithms: Algorithm 1 gives estimated piecewise linear distortions to fit a given data, and Algorithm 2 gives smoothed version of these distortions by using hyperbolic compositions in the logit scale.

The theoretical properties of considered distortions are thus very helpful for fitting distorted multivariate distributions. Applications on real data have emphasis the advantages of this methodology. First, despite the variable and possibly high number of parameters, the estimation is straightforward and only relying on univariate optimizations. Second, the methodology benefit from the theoretical (and practical) possibility to fit marginal distributions as precisely as desired. Third, it leads to parametric expressions of both cumulative distribution functions and level curves, which can be useful for risk measures. Fourth, numerical results on real data easily lead to a better likelihood than other parametric representations of the same data in some recent studies.

Some interesting perspectives would be a better characterization of necessary and sufficient conditions for regular external distortions, using the extreme value theory to estimate these multivariate quantile curves in order to improve the estimation quality for large values of $\alpha$ (see Remark 9), some work on the optimal initial levels to be chosen for the proposed algorithms, and on how to choose or reduce the number of parameters. The use of the parametric level curves for risk measures also opens a large research field.

Acknowledgements: The authors thank an anonymous referee for constructive remarks and valuable suggestions to improve the paper. This work has been partially supported by the French National Research Agency (ANR) under the reference ANR-08BLAN-0314-01. Part of this work also benefit from the support of the MIRACCLE-GICC project.

\section{References}

[1] G. Awanou, M. Lai, and P. Wenston. The multivariate spline method for scattered data fitting and numerical solutions of partial differential equations. Wavelets and Splines: Athens, pages 24-74, 2005.

[2] F. Belzunce, A. Castaño, A. Olvera-Cervantes, and A. Suárez-Llorens. Quantile curves and dependence structure for bivariate distributions. Computational Statistics \& Data Analysis, 51(10):5112-5129, 2007.

[3] A. Bienvenüe and D. Rullière. Iterative adjustment of survival functions by composed probability distortions. The Geneva Risk and Insurance Review, 37(2):156-179, 2011.

[4] A. Bienvenüe and D. Rullière. On hyperbolic iterated distortions for the adjustment of survival functions. In C. Perna and M. Sibillo, editors, Mathematical and Statistical Methods for Actuarial Sciences and Finance, pages 35-42. Springer Milan, 2012.

[5] C. Biernacki, G. Celeux, and G. Govaert. Choosing starting values for the EM algorithm for getting the highest likelihood in multivariate Gaussian mixture models. Comput. Statist. Data Anal., 41(3-4):561-575, 2003. Recent developments in mixture models (Hamburg, 2001).

[6] J. E. Chacon and A. Rodriguez-Casal. A note on the universal consistency of the kernel distribution function estimator. Statistics $\&$ Probability Letters, 80(17-18):1414-1419, 2010.

[7] A. Charpentier, F. J.D., and O. Scaillet. The estimation of copulas: theory and practice. In Copulas: from theory to application in finance. Risk Books; 1 edition, 2006.

[8] F. Chebana and T. Ouarda. Multivariate quantiles in hydrological frequency analysis. Environmetrics, 22(1):63-78, 2011.

[9] A. Cousin and E. Di Bernardino. On multivariate extensions of Value-at-Risk. Submitted, 2013.

[10] L. de Haan and X. Huang. Large quantile estimation in a multivariate setting. J. Multivariate Anal., 53(2):247-263, 1995. 
[11] E. Di Bernardino, T. Laloë, V. Maume-Deschamps, and C. Prieur. Plug-in estimation of level sets in a non-compact setting with applications in multivariable risk theory. ESAIM: Probability and Statistics, http://dx.doi.org/10.1051/ps/2011161, 2011.

[12] F. Durante, R. Foschi, and P. Sarkoci. Distorted copulas: Constructions and tail dependence. Communications in Statistics - Theory and Methods, 39(12):2288-2301, 2010.

[13] V. Durrleman, A. Nikeghbali, and T. Roncalli. A simple transformation of copulas. Technical report, Groupe de Research Operationnelle Credit Lyonnais, 2000.

[14] P. Embrechts and G. Puccetti. Bounds for functions of multivariate risks. Journal of Multivariate Analysis, 97(2):526$547,2006$.

[15] E. W. Frees and E. A. Valdez. Understanding relationships using copulas. North American Actuarial Journal, 2(1):1-25, 1998.

[16] S. A. Klugman and R. Parsa. Fitting bivariate loss distributions with copulas. Insurance Math. Econom., 24(1-2):139148, 1999. 1st IME Conference (Amsterdam, 1997).

[17] A. McNeil, R. Frey, and P. Embrechts. Quantitative Risk Management: Concepts, Techniques, and Tools. Princeton Series in Finance. Princeton University Press, 2005.

[18] A. McNeil and J. Nešlehová. Multivariate archimedean copulas, d-monotone functions and $l_{1}-$ norm symmetric distributions. The Annals of Statistics, 37(5B):3059-3097, 2009.

[19] G. Nappo and F. Spizzichino. Kendall distributions and level sets in bivariate exchangeable survival models. Information Sciences, 179:2878-2890, August 2009.

[20] R. B. Nelsen. An introduction to copulas, volume 139 of Lecture Notes in Statistics. Springer-Verlag, New York, 1999.

[21] A. Obereder, O. Scherzer, and A. Kovac. Bivariate density estimation using BV regularisation. Comput. Statist. Data Anal., 51(12):5622-5634, 2007.

[22] C. Rossi. Sulle curve di livello di una superficie di ripartizione in due variabili. Giornale dell'Istituto Italiano degli Attuari, 36:87-108, 1973.

[23] G. Salvadori, C. De Michele, N. Kottegoda, and R. Rosso. Extremes in Nature: An Approach Using Copulas. SpringerVerlag: Berlin, 2007.

[24] L. Tibiletti. Gli insiemi di livello delle funzioni di ripartizione n-dimensionali: un'applicazione statistica (Level sets of n-dimensional distribution functions: a statistical application). Quaderni dell'Istituto di Matematica Finanziaria dell'universit di Torino: terza serie, 56:1-11, 1990.

[25] L. Tibiletti. Sulla quasi concavita delle funzioni di ripartizione n-dimensionali - on quasi-concavity of n-dimensional distribution functions. In Atti del XV convegno A.M.A.S.E.S., pages 503-515, 1991.

[26] L. Tibiletti. On a new notion of multidimensional quantile. Metron. International Journal of Statistics, 51(3-4):77-83, 1993.

[27] E. A. Valdez and Y. X. On the distortion of a copula and its margins. Scandinavian Actuarial Journal, 2011. accepted for publication and forthcoming. 